\title{
UPPER AND LOWER SEMICONTINUITY OF IMPULSIVE COCYCLE ATTRACTORS FOR IMPULSIVE NONAUTONOMOUS SYSTEMS
}

\author{
E. M. Bonotto ${ }^{1}$, M. C. Bortolan ${ }^{2}$, T. $_{\text {Caraballo }}^{3} \&$ R. Collegari ${ }^{4}$
}

\begin{abstract}
In this work we present results to ensure a weak upper semicontinuity for a family of impulsive cocycle attractors of nonautonomous impulsive dynamical systems, as well as an example of nonautonomous dynamical system generated by an ODE in the real line to illustrate our results. Moreover, we present theoretical results regarding lower semicontinuity of impulsive cocycle attractors.
\end{abstract}

Keywords: weak upper semicontinuity; weak lower semicontinuity, continuity, nonautonomous impulsive dynamical system; impulsive cocycle attractor, collective tube conditions.

2010 Mathematics Subject Classification: Primary 35B41. Secondary 34A37; 35R12.

\section{Contents}

1. Introduction 2

2. Impulsive nonautonomous dynamical systems 3

2.1. Tube conditions 6

\begin{tabular}{lll}
\hline 2.2. & Existence of impulsive cocycle attractors & 7
\end{tabular}

3. Collective tube conditions for INDS 9

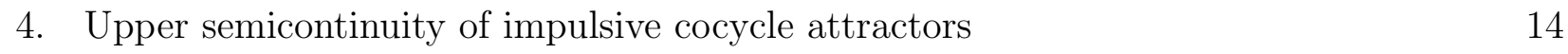

$\begin{array}{lll}5 . & \text { Applications } & 21\end{array}$

\begin{tabular}{|ll}
\hline 6. & Lower semicontinuity \\
\hline
\end{tabular}

\begin{tabular}{ll}
\hline Conclusion and final remarks & 27 \\
\hline
\end{tabular}

\begin{tabular}{ll}
\hline References & 27
\end{tabular}

Partially supported by: ${ }^{1}$ FAPESP (2016/24711-1) and CNPq (310497/2016-7), Brazil. ${ }^{2}$ CNPq (407635/20165), Brazil. ${ }^{3}$ FEDER and Ministerio de Economía y Competitividad (MTM2011-22411) and Consejería de Innovación, Ciencia y Empresa (Junta de Andalucía) under Proyecto de Excelencia P12-FQM-1492, Spain. ${ }^{4}$ FAPESP (2014/20691-0), Brazil. 


\section{Introduction}

When dealing with the modeling of real world phenomena, perturbations are likely to occur. Truncated measurements in data collection, simplifications in the model, and even the use of well known empirical laws, are bound to introduce particularities in the modeled problem that do not appear in the real one. These small divergences are expected to occur, but if left untreated they may invalidate the obtained results when we want to translate them to the real problem. How can we be sure that the obtained properties may be transported to the limiting problem without any loss of information? Or if there is some kind of loss, can we foresee how small or how large will it be?

This problem can be treated in several different ways and in a few distinct levels of complexity. The first level is the upper semicontinuity, which roughly speaking means that we can assure that the solutions of the perturbed problems are close to the solutions of the limiting problem, as long as the perturbation is small and well behaved. Formally speaking, a family $\left\{A_{\lambda}\right\}_{\lambda \in \Lambda}$ of nonempty subsets of a metric space $(X, d)$, indexed in a topological space $\Lambda$, is said to be upper semicontinuous at $\lambda_{0}$ if

$$
\lim _{\lambda \rightarrow \lambda_{0}} \mathrm{~d}_{H}\left(A_{\lambda}, A_{\lambda_{0}}\right)=0
$$

where $\mathrm{d}_{H}(A, B)=\sup _{a \in A} \inf _{b \in B} d(a, b)$ is the Hausdorff semidistance between $A$ and $B$. This semidistance measures how far $A$ is from being inside the closure of $B$. Hence, in such a way, the upper semicontinuity at $\lambda_{0}$ means that $A_{\lambda}$ is not far from being inside $\overline{A_{\lambda_{0}}}$, for $\lambda$ sufficiently close to $\lambda_{0}$.

This matter, as well as the more complicated problem of lower semicontinuity, is well established for the case of continuous dynamical systems both in the autonomous and nonautonomous framework (see, for instance, [2, 17, 11, 16]). The parallel in the impulsive case has seen its first steps very recently in [4] for the autonomous case. For nonautonomous impulsive dynamical systems we are not aware of results in this line and we intend to present some results in this work.

The next level in the study of continuity is the already mentioned lower semicontinuity, and it is related to the non-implosion of solutions of the perturbed problems, for small and well behaved perturbations. The family $\left\{A_{\lambda}\right\}_{\lambda \in \Lambda}$ is said to be lower semicontinuous at $\lambda_{0}$ if

$$
\lim _{\lambda \rightarrow \lambda_{0}} \mathrm{~d}_{H}\left(A_{\lambda_{0}}, A_{\lambda}\right)=0
$$

which can be translated to the property that, for $\lambda$ close to $\lambda_{0}$, the sets $A_{\lambda}$ are as complex as $A_{\lambda_{0}}$. Although it is just a change of position in the computation of the Hausdorff semidistance, the lower semicontinuity if a far more difficult subject than the upper semicontinuity, and to be tackled, it requires a knowledge of the internal structures of $A_{\lambda_{0}}$. 
Nevertheless, the lower semicontinuity has also been studied in the past decades and is still being developed nowdays. Both theoretical abstract results and their applications to attractors of continuous differential equations can be seen in [8, 11, 16, 17]. Again, the parallel in the impulsive autonomous case was developed in [4], where the authors presented abstract results to obtain lower semicontinuity. There are also higher levels of continuity, namely the topological stability and the geometrical stability, which will not be discussed here, but in forthcoming works.

In what follows, Section 2 is an overview of the already known results concerning the existence of impulsive cocycle attractors for impulsive dynamical systems. For a more detailed approach, the reader may see [3, 5. In this section, we will also set the main definitions and notations that will be used throughout the paper. In Section 3, we present the so called collective tube conditions for impulsive nonautonomous dynamical systems (see Definitions 3.1 and 3.2. Such conditions play a crucial role when dealing with perturbations of impulsive systems, and the definitions presented here follow the ideas contained in [4] for the autonomous case, which are briefly described in Subsection 2.1. We also present two new results (see Lemma 3.10 and Theorem 3.12 that will be used to prove the upper semicontinuity of impulsive cocycle attractors in Section 4 .

Section 4 is the main section of this work and contains a result presenting sufficient conditions to ensure the upper semicontinuity for a family of impulsive cocycle attractors for impulsive nonautonomous dynamical systems (see Theorem 4.4). Furthermore, we present an example of an impulsive nonautonomous dynamical system in the real line to illustrate our main result (see Section 5).

In Section 6, we present a theoretical approach to the results regarding lower semicontinuity and we end our paper with a final section containing the summary of our results and some open problems of this theory.

\section{Impulsive nonautonomous dynamical systems}

The theory of continuous nonautonomous dynamical systems is very well established and here, to make our presentation as much self-contained as possible, we will present the main concepts concerning impulsive nonautonomous dynamical systems. For more details on the continuous theory, the reader may consult $[1,6, \mathbf{6}, \mathbf{1 0}, \mathbf{1 2}, 13,18$.

Let $\mathbb{R}_{+}=[0,+\infty)$ and $\mathbb{N}=\{1,2,3, \ldots\}$ be the set of all natural numbers. We denote $\mathbb{N}_{0}$ by $\mathbb{N} \cup\{0\}$. We will denote, in any space, sequences indexed in $\mathbb{N}$ by $\left\{a_{k}\right\}$ or $\left\{a_{k}\right\}_{k}$, and the latter will be used if it is necessary to avoid confusion with the index.

Let $\Sigma$ be a complete metric space and $\left\{\theta_{t}: t \geqslant 0\right\}$ be a semigroup in $\Sigma$, that is, it is a family of continuous maps from $\Sigma$ into itself, satisfying the following conditions: $\theta_{0} \sigma=\sigma$ for all $\sigma \in \Sigma$, $\theta_{t+s}=\theta_{t} \theta_{s}$ for all $t, s \in \mathbb{R}_{+}$and the map $\mathbb{R}_{+} \times \Sigma \ni(t, \sigma) \mapsto \theta_{t} \sigma \in \Sigma$ is continuous. 
Also, let us consider another complete metric space $(X, d)$ and for each pair $(t, \sigma) \in \mathbb{R}_{+} \times \Sigma$, let $\varphi(t, \sigma): X \rightarrow X$ be a map satisfying the following properties:

(i) $\varphi(0, \sigma) x=x$ for all $x \in X$ and $\sigma \in \Sigma$;

(ii) $\varphi(t+s, \sigma)=\varphi\left(t, \theta_{s} \sigma\right) \varphi(s, \sigma)$ for all $t, s \in \mathbb{R}_{+}$and $\sigma \in \Sigma$;

(iii) the map $\mathbb{R}_{+} \times \Sigma \times X \ni(t, \sigma, x) \mapsto \varphi(t, \sigma) x \in X$ is continuous.

Definition 2.1. With the previous definitions and relations, $(\varphi, \theta)_{(X, \Sigma)}$ is said to be a nonautonomous dynamical system, or simply a NDS.

The semigroup $\left\{\theta_{t}: t \geqslant 0\right\}$ in this context is called driving semigroup, the map $\varphi$ is called cocycle and the property (ii) above is commonly known as the cocycle property.

A nonautonomous set is a family $\hat{D}=\{D(\sigma)\}_{\sigma \in \Sigma}$ of subsets of $X$ indexed in $\Sigma$. We say that $\hat{D}$ is an open (closed, compact) nonautonomous set if each fiber $D(\sigma)$ is an open (closed, compact) subset of $X$. A nonautonomous set $\hat{D}$ is called $\varphi$-invariant if

$$
\varphi(t, \sigma) D(\sigma)=D\left(\theta_{t} \sigma\right) \text { for all } t \geqslant 0 \text { and each } \sigma \in \Sigma .
$$

A universe $\mathfrak{D}$ is a collection of nonautonomous sets which is closed with respect to inclusion, that is, if $\hat{D}_{1} \in \mathfrak{D}$ and $D_{2}(\sigma) \subset D_{1}(\sigma)$ for all $\sigma \in \Sigma$, then $\hat{D}_{2} \in \mathfrak{D}$.

In the sequel, we present the concept of impulsive nonautonomous dynamical systems.

Let $(\varphi, \theta)_{(X, \Sigma)}$ be a NDS and for each $D \subset X, J \subset \mathbb{R}_{+}$and $\sigma \in \Sigma$, we define

$$
F_{\varphi}(D, J, \sigma)=\{x \in X: \varphi(t, \sigma) x \in D \text { for some } t \in J\} \text {. }
$$

A point $x \in X$ is said to be an initial point if $F_{\varphi}(x, \tau, \sigma)=\varnothing$ for all $\tau>0$ and for all $\sigma \in \Sigma$.

Definition 2.2. An impulsive nonautonomous dynamical system, or simply an INDS, denoted by $\left[(\varphi, \theta)_{(X, \Sigma)}, M, I\right]$, consists of a $\operatorname{NDS}(\varphi, \theta)_{(X, \Sigma)}$, a nonempty closed subset $M \subset X$ such that for each $x \in M$ and each $\sigma \in \Sigma$ there exists $\epsilon_{x, \sigma}>0$ such that

$$
\bigcup_{t \in\left(0, \epsilon_{x, \sigma}\right)}\left(F_{\varphi}\left(x, t, \theta_{-t} \sigma\right) \bigcap M\right)=\varnothing \quad \text { and } \quad\left\{\varphi(s, \sigma) x: s \in\left(0, \epsilon_{x, \sigma}\right)\right\} \bigcap M=\varnothing
$$

and a continuous function $I: M \rightarrow X$ whose action will be specified later. The set $M$ is called the impulsive set and the function $I$ is called the impulse function. We also define $M_{\varphi}^{+}(x, \sigma)=\{\varphi(\tau, \sigma) x: \tau>0\} \bigcap M$ for each $(x, \sigma) \in X \times \Sigma$.

One important property which may be observed is that if $M_{\varphi}^{+}(x, \sigma) \neq \varnothing$, then there exists $t>0$ such that $\varphi(t, \sigma) x \in M$ and $\varphi(\tau, \sigma) x \notin M$ for $0<\tau<t$, and as a consequence, for each $\sigma \in \Sigma$, we are able to define the function $\phi(\cdot, \sigma): X \rightarrow(0,+\infty]$ by

$$
\phi(x, \sigma)= \begin{cases}s, & \text { if } \varphi(s, \sigma) x \in M \text { and } \varphi(t, \sigma) x \notin M \text { for } 0<t<s, \\ +\infty, & \text { if } \varphi(t, \sigma) x \notin M \text { for all } t>0 .\end{cases}
$$


In the first case, the value $\phi(x, \sigma)$ represents the smallest positive time such that the positive semitrajectory of $x$ in the fiber $\sigma$ meets $M$ and we say that the point $\varphi(\phi(x, \sigma), \sigma) x$ is the impulsive point of $x$ in the fiber $\sigma$.

Definition 2.3. Given $\sigma \in \Sigma$, the impulsive positive semitrajectory of $x \in X$ starting at $\sigma$ by the INDS $\left[(\varphi, \theta)_{(X, \Sigma)}, M, I\right]$ is a map $\tilde{\varphi}(\cdot, \sigma) x$ defined in an interval $J_{(x, \sigma)} \subset \mathbb{R}_{+}$, $0 \in J_{(x, \sigma)}$, with values in $X$ given inductively by the following rule: if $M_{\varphi}^{+}(x, \sigma)=\varnothing$, then $\tilde{\varphi}(t, \sigma) x=\varphi(t, \sigma) x$ for all $t \in[0,+\infty)$ and in this case $\phi(x, \sigma)=+\infty$. However, if $M_{\varphi}^{+}(x, \sigma) \neq \varnothing$ then we denote $x=x_{0}^{+}$and we define $\tilde{\varphi}(\cdot, \sigma) x$ on $\left[0, \phi\left(x_{0}^{+}, \sigma\right)\right]$ by

$$
\tilde{\varphi}(t, \sigma) x= \begin{cases}\varphi(t, \sigma) x_{0}^{+}, & \text {if } \quad 0 \leqslant t<\phi\left(x_{0}^{+}, \sigma\right), \\ I\left(\varphi\left(\phi\left(x_{0}^{+}, \sigma\right), \sigma\right) x_{0}^{+}\right), & \text {if } \quad t=\phi\left(x_{0}^{+}, \sigma\right) .\end{cases}
$$

Now, let $s_{0}=\phi\left(x_{0}^{+}, \sigma\right), x_{1}=\varphi\left(s_{0}, \sigma\right) x_{0}^{+}$and $x_{1}^{+}=I\left(\varphi\left(s_{0}, \sigma\right) x_{0}^{+}\right)$. In this case, $s_{0}<+\infty$ and the process can go on, but now starting at $x_{1}^{+}$. If $M_{\varphi}^{+}\left(x_{1}^{+}, \theta_{s_{0}} \sigma\right)=\varnothing$ then we define $\tilde{\varphi}(t, \sigma) x=\varphi\left(t-s_{0}, \theta_{s_{0}} \sigma\right) x_{1}^{+}$for $s_{0} \leqslant t<+\infty$ and in this case $\phi\left(x_{1}^{+}, \theta_{s_{0}} \sigma\right)=+\infty$. However, if $M_{\varphi}^{+}\left(x_{1}^{+}, \theta_{s_{0}} \sigma\right) \neq \varnothing$, then we define $\tilde{\varphi}(\cdot, \sigma) x$ on $\left[s_{0}, s_{0}+\phi\left(x_{1}^{+}, \theta_{s_{0}} \sigma\right)\right]$ by

$$
\tilde{\varphi}(t, \sigma) x=\left\{\begin{array}{lll}
\varphi\left(t-s_{0}, \theta_{s_{0}} \sigma\right) x_{1}^{+}, & \text {if } \quad s_{0} \leqslant t<s_{0}+\phi\left(x_{1}^{+}, \theta_{s_{0}} \sigma\right), \\
I\left(\varphi\left(\phi\left(x_{1}^{+}, \theta_{s_{0}} \sigma\right), \theta_{s_{0}} \sigma\right) x_{1}^{+}\right), & \text {if } \quad t=s_{0}+\phi\left(x_{1}^{+}, \theta_{s_{0}} \sigma\right) .
\end{array}\right.
$$

Now let $s_{1}=\phi\left(x_{1}^{+}, \theta_{s_{0}} \sigma\right), x_{2}=\varphi\left(s_{1}, \theta_{s_{0}} \sigma\right) x_{1}^{+}$and $x_{2}^{+}=I\left(\varphi\left(s_{1}, \theta_{s_{0}} \sigma\right) x_{1}^{+}\right)$, and so on.

This process ends after a finite number of steps if $M_{\varphi}^{+}\left(x_{n}^{+}, \theta_{t_{n}} \sigma\right)=\varnothing$ for some $n \geqslant 0$ (with $t_{0}=0$ and $t_{n}=s_{0}+\ldots+s_{n-1}$ for $\left.n \geqslant 1\right)$, or it may proceed indefinitely, if $M_{\varphi}^{+}\left(x_{n}^{+}, \theta_{t_{n}} \sigma\right) \neq \varnothing$ for all $n \geqslant 0$, and in this case $\tilde{\varphi}(\cdot, \sigma) x$ is defined on the interval $[0, T(x, \sigma))$, where $T(x, \sigma)=\sum_{i=0}^{+\infty} s_{i}$.

As presented in [3], we may assume hereon the following assumption:

$$
T(x, \sigma)=+\infty \quad \text { for all } \quad x \in X \text { and } \sigma \in \Sigma \text {. }
$$

This assumption holds, for instance, when there exists $\xi>0$ such that $\phi(x, \sigma) \geqslant \xi$ for all $x \in I(M)$ and $\sigma \in \Sigma$.

Definition 2.4. Given $x \in X$ and $\sigma \in \Sigma$, a time $t \geqslant 0$ is called an impulsive time for $\tilde{\varphi}(\cdot, \sigma) x$ if there is $n \geqslant 0$ such that $t=\sum_{i=0}^{n} s_{i}$, where $s_{0}=\phi(x, \sigma)$ and $s_{i}=\phi\left(x_{i}^{+}, \theta_{t_{i}} \sigma\right)$ with $t_{i}=s_{0}+\ldots+s_{i-1}, i=1,2, \ldots n$.

Remark 2.5. The construction of the function $\phi$ and the impulsive positive semitrajectory $\tilde{\varphi}$ allows us to state the following important relationship, whose proof may be found in [3]. Let 
$(\varphi, \theta)_{(X, \Sigma)}$ be a NDS and $\{\Pi(t): t \geqslant 0\}$ be its associated skew-product semiflow in $\mathbb{X}=X \times \Sigma$. Define $\tilde{\Pi}^{*}$ by

$$
\tilde{\Pi}^{*}(t)(x, \sigma)=\left(\tilde{\varphi}(t, \sigma) x, \theta_{t} \sigma\right) \quad \text { for all } \quad(x, \sigma) \in \mathbb{X} \text { and } t \geqslant 0,
$$

and also let $\{\tilde{\Pi}(t): t \geqslant 0\}$ be the impulsive dynamical system $(\mathbb{X}, \Pi, \mathbb{M}, \mathbb{I})$, where $\mathbb{M}=M \times \Sigma$ and $\mathbb{I}: \mathbb{M} \rightarrow \mathbb{X}$ is given by $\mathbb{I}(x, \sigma)=(I(x), \sigma)$, for $x \in M$ and $\sigma \in \Sigma$. Then

$$
\tilde{\Pi}^{*}(t)=\tilde{\Pi}(t) \quad \text { for all } \quad t \geqslant 0 .
$$

Moreover, if $\phi$ is the function defined in (2.2), then it coincides with the function used to define the impulsive positive semitrajectory $\{\tilde{\Pi}(t): t \geqslant 0\}$. Also, for each $\sigma \in \Sigma$ and $t, s \in \mathbb{R}_{+}$, we have

$$
\tilde{\varphi}(t+s, \sigma)=\tilde{\varphi}\left(t, \theta_{s} \sigma\right) \tilde{\varphi}(s, \sigma),
$$

that is, $\tilde{\varphi}$ satisfies the cocycle property.

2.1. Tube conditions. The tube conditions are very important for the theory of impulsive dynamical systems. Here, we briefly present the results of [3] (which uses the results of [15]) for tube conditions of impulsive nonautonomous dynamical systems. Recall that $\mathbb{X}=X \times \Sigma$ and $\mathbb{M}=M \times \Sigma$. For $\mathbb{D} \subset \mathbb{X}$ and $J \subset \mathbb{R}_{+}$we define

$$
\mathbb{F}(\mathbb{D}, J)=\{(x, \sigma) \in \mathbb{X}: \Pi(t)(x, \sigma) \in \mathbb{D} \text { for some } t \in J\} .
$$

Definition 2.6. A closed set $\mathbb{S}$ containing $(x, \sigma) \in \mathbb{X}$ is called a section through $(x, \sigma)$ if there exist $\lambda>0$ and a closed subset $\mathbb{L}$ of $\mathbb{X}$ such that:

(a) $\mathbb{F}(\mathbb{L}, \lambda)=\mathbb{S}$;

(b) $\mathbb{F}(\mathbb{L},[0,2 \lambda])$ contains a neighborhood of $(x, \sigma)$;

(c) $\mathbb{F}(\mathbb{L}, \nu) \bigcap \mathbb{F}(\mathbb{L}, \zeta)=\varnothing$, if $0 \leqslant \nu<\zeta \leqslant 2 \lambda$.

We say that the set $\mathbb{F}(\mathbb{L},[0,2 \lambda])$ is a $\lambda$-tube (or simply tube) and the set $\mathbb{L}$ is a bar.

The Definition 2.6 is the same definition of tube for general impulsive systems $(X, \pi, M, I)$, see [14].

Definition 2.7. A point $(x, \sigma) \in \mathbb{M}$ satisfies the strong tube condition (STC), if there exists a section $\mathbb{S}$ through $(x, \sigma)$ such that $\mathbb{S}=\mathbb{F}(\mathbb{L},[0,2 \lambda]) \bigcap \mathbb{M}$. Also, we say that a point $(x, \sigma) \in \mathbb{M}$ satisfies the special strong tube condition (SSTC) if it satisfies STC and the $\lambda$-tube $\mathbb{F}(\mathbb{L},[0,2 \lambda])$ is such that $\mathbb{F}(\mathbb{L},[0, \lambda]) \bigcap \mathbb{I}(\mathbb{M})=\varnothing$.

Now, we introduce the concepts of STC and SSTC in the context of INDS.

Definition 2.8. Let $\left[(\varphi, \theta)_{(X, \Sigma)}, M, I\right]$ be an INDS. We say that a point $x \in M$ satisfies the $\varphi$-strong tube condition ( $\varphi$-STC), if for each $\sigma \in \Sigma$, the pair $(x, \sigma)$ satisfies STC with respect to the impulsive skew-product $(\mathbb{X}, \Pi, \mathbb{M}, \mathbb{I})$. Also, we say that a point $x \in M$ satisfies the $\varphi$-special strong tube condition ( $\varphi$-SSTC), if for each $\sigma \in \Sigma$, the pair $(x, \sigma)$ satisfies SSTC with respect to the impulsive skew-product $(\mathbb{X}, \Pi, \mathbb{M}, \mathbb{I})$. 
Next, we exhibit two auxiliary results.

Theorem 2.9. [3, Theorem 3.5] Let $\left[(\varphi, \theta)_{(X, \Sigma)}, M, I\right]$ be an INDS such that each point of $M$ satisfies $\varphi-S T C$. Then $\phi$ is upper semicontinuous in $X \times \Sigma$, and it is continuous in $(X \backslash M) \times \Sigma$. Moreover, if there are no initial points in $M$ and $\phi$ is continuous at $(x, \sigma)$ for some $\sigma \in \Sigma$, then $x \notin M$.

Proposition 2.10. [3. Propositon 3.7] Let $\left[(\varphi, \theta)_{(X, \Sigma)}, M, I\right]$ be an INDS such that $I(M) \bigcap M=$ $\varnothing$ and let $y \in M$ satisfy $\varphi$-SSTC. Then, for each $\sigma \in \Sigma$, the point $(y, \sigma)$ satisfies SSTC with $\lambda$-tube $\mathbb{F}(\mathbb{L},[0,2 \lambda])$ such that $\tilde{\Pi}(t) \mathbb{X} \cap \mathbb{F}(\mathbb{L},[0, \lambda])=\varnothing$ for all $t>\lambda$.

This part of the paper demands a small explanation. There is a question on why we do not define the tube conditions (and then the collective tube conditions in Section 3) using directly the nonautonomous dynamical system $\left[(\varphi, \theta)_{(X, \Sigma)}, M, I\right]$ instead of the associated impulsive skew-product $(\mathbb{X}, \Pi, \mathbb{M}, \mathbb{I})$. One short answer to this problem is that "because it works when we use the impulsive skew-product", but of course this is not a mathematic response. The main problem there is that in the nonautonomous context, we are constantly changing the fibers when using $\varphi(t, \sigma)$ - we go from $\sigma$ to $\theta_{t} \sigma$ - and therefore the continuity required to prove Theorem 2.9, does not work properly without the second coordinate of the skew-product semiflow. Hence, it is not possible to ensure the continuity of $\phi$ (even only for the first variable), which is paramount, using only properties of the impulsive nonautonomous dynamical system instead the ones of the impulsive skew-product.

2.2. Existence of impulsive cocycle attractors. In [3], the definition of impulsive nonautonomous dynamical systems is introduced and also sufficient conditions to ensure the existence of an impulsive cocycle attractor are proved. In this subsection, we present their main results.

The definition of $\tilde{\varphi}$-invariance is analogous to the notion of $\varphi$-invariance simply replacing $\varphi$ by $\tilde{\varphi}$.

Definition 2.11. Given an $\operatorname{INDS}\left[(\varphi, \theta)_{(X, \Sigma)}, M, I\right]$, a nonautonomous set $\hat{B}$ is said to be pullback $(\tilde{\varphi}, \mathfrak{D})$-attracting, if for each $\sigma \in \Sigma$ and $\hat{D} \in \mathfrak{D}$ we have

$$
\lim _{t \rightarrow+\infty} \mathrm{d}_{H}\left(\tilde{\varphi}\left(t, \theta_{-t} \sigma\right) D\left(\theta_{-t} \sigma\right), B(\sigma)\right)=0,
$$

where $\mathrm{d}_{H}(\cdot, \cdot)$ denotes the Hausdorff semidistance, that is, $\mathrm{d}_{H}(C, D)=\sup _{x \in C} \inf _{y \in D} d(x, y)$ for any nonempty sets $C, D \subset X$.

A nonautonomous set $\hat{B}$ is said to be pullback $\mathfrak{D}$-absorbing for the $\operatorname{INDS}\left[(\varphi, \theta)_{(X, \Sigma)}, M, I\right]$, if for each $\sigma \in \Sigma$ and $\hat{D} \in \mathfrak{D}$, there exists $t_{0}=t_{0}(\sigma, \hat{D}) \geqslant 0$ such that

$$
\tilde{\varphi}\left(t, \theta_{-t} \sigma\right) D\left(\theta_{-t} \sigma\right) \subseteq B(\sigma) \text { for all } t \geqslant t_{0} .
$$

Definition 2.12. Given a universe $\mathfrak{D}$ and an INDS $\left[(\varphi, \theta)_{(X, \Sigma)}, M, I\right]$, a compact nonautonomous set $\hat{A}$ is called the $(\tilde{\varphi}, \mathfrak{D})$-impulsive cocycle attractor if: 
(i) $\hat{A} \backslash M=\{A(\sigma) \backslash M\}_{\sigma \in \Sigma}$ is $\tilde{\varphi}$-invariant;

(ii) $\hat{A}$ is pullback $(\tilde{\varphi}, \mathfrak{D})$-attracting;

(iii) $\hat{A}$ is minimal among the closed nonautonomous sets satisfying (ii).

We may observe that if $\hat{A}_{1}$ and $\hat{A}_{2}$ are two $(\tilde{\varphi}, \mathfrak{D})$-impulsive cocycle attractors then $A_{1}(\sigma) \backslash M=A_{2}(\sigma) \backslash M$ for all $\sigma \in \Sigma$.

As studied in [5], to find sufficient conditions to ensure the existence of an impulsive cocycle attractor for an INDS, we present at first the concept of the impulsive pullback $\omega$-limit set.

Definition 2.13. Given a universe $\mathfrak{D}$, a nonautonomous set $\hat{B} \in \mathfrak{D}$ and $\sigma \in \Sigma$, we define the impulsive pullback $\omega$-limit of $\hat{B}$ at $\sigma$ as the set

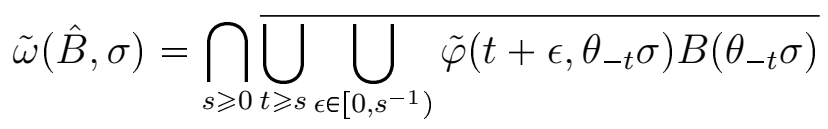

and the impulsive pullback $\omega$-limit of $\hat{B}$ as the nonautonomous set $\tilde{\omega}(\hat{B})=\{\tilde{\omega}(\hat{B}, \sigma)\}_{\sigma \in \Sigma}$.

The impulsive pullback $\omega$-limit set also may be characterized as presented in the next result.

Lemma 2.14. [3, Lemma 4.2] For $\hat{B} \in \mathfrak{D}$ and $\sigma \in \Sigma$, we have

$$
\begin{array}{r}
\tilde{\omega}(\hat{B}, \sigma)=\left\{x \in X: \text { there exist sequences }\left\{t_{n}\right\}_{n \geqslant 1},\left\{\epsilon_{n}\right\}_{n \geqslant 1} \subseteq \mathbb{R}_{+} \text {and }\left\{x_{n}\right\}_{n \geqslant 1} \subseteq B\left(\theta_{-t_{n}} \sigma\right)\right. \\
\text { with } \left.t_{n} \stackrel{n \rightarrow \infty}{\longrightarrow} \infty, \epsilon_{n} \stackrel{n \rightarrow \infty}{\longrightarrow} 0 \text { such that } \tilde{\varphi}\left(t_{n}+\epsilon_{n}, \theta_{-t_{n}} \sigma\right) x_{n} \stackrel{n \rightarrow \infty}{\longrightarrow} x\right\}
\end{array}
$$

and $\tilde{\omega}(\hat{B}, \sigma)$ is closed in $X$.

Next, we present the concept of pullback $\mathfrak{D}$-asymptotically compactness for INDS.

Definition 2.15. An INDS $\left[(\varphi, \theta)_{(X, \Sigma)}, M, I\right]$ is said to be pullback $\mathfrak{D}$-asymptotically compact, if for any $\sigma \in \Sigma, \hat{D} \in \mathfrak{D}$ and sequences $\left\{t_{n}\right\}_{n \geqslant 1} \subset \mathbb{R}_{+},\left\{x_{n}\right\}_{n \geqslant 1} \subset X$ with $t_{n} \stackrel{n \rightarrow+\infty}{\longrightarrow}+\infty$ and $x_{n} \in D\left(\theta_{-t_{n}} \sigma\right)$ for $n \geqslant 1$, then the sequence $\left\{\tilde{\varphi}\left(t_{n}, \theta_{-t_{n}} \sigma\right) x_{n}\right\}_{n \geqslant 1}$ possesses a convergent subsequence.

The existence of the $(\tilde{\varphi}, \mathfrak{D})$-impulsive cocycle attractor is presented in Theorem 2.16 .

Theorem 2.16. [5, Theorem 3.16] Let $\left[(\varphi, \theta)_{(X, \Sigma)}, M, I\right]$ be an INDS pullback $\mathfrak{D}$-asymptotically compact such that $I(M) \bigcap M=\varnothing$ and every point from $M$ satisfies $\varphi-S S T C$. Assume that there exists a pullback $(\tilde{\varphi}, \mathfrak{D})$-attracting nonautonomous set $\hat{K} \in \mathfrak{D}$. Then, the nonautonomous set $\hat{A}$, given by $A(\sigma)=\tilde{\omega}(\hat{K}, \sigma)$, is the $(\tilde{\varphi}, \mathfrak{D})$-impulsive cocycle attractor.

Definition 2.17. Given an INDS $\left[(\varphi, \theta)_{(X, \Sigma)}, M, I\right]$, we say that $\psi: \mathbb{R} \rightarrow X$ is a global solution of $\tilde{\varphi}$ at $\sigma \in \Sigma$ if

$$
\tilde{\varphi}\left(t-s, \theta_{s} \sigma\right) \psi(s)=\psi(t) \text { for all } t \geqslant s \text { and } s \in \mathbb{R} .
$$

If $\psi(0)=x$ then $\psi$ is called a global solution of $\tilde{\varphi}$ through $x$ at $\sigma$. If $\psi(\mathbb{R})$ is a bounded subset of $X$, we say that $\psi$ is a bounded global solution of $\tilde{\varphi}$. 
Corollary 2.18. [3, Proposition 5.5] If the $\operatorname{INDS}\left[(\varphi, \theta)_{(X, \Sigma)}, M, I\right]$ has an impulsive cocycle attractor $\hat{A} \in \mathfrak{D}$ with universe $\mathfrak{D}$ consisting of all the nonautonomous sets $\hat{B}$ such that $\bigcup_{\sigma \in \Sigma} B(\sigma)$ is bounded in $X$ and $I(M) \bigcap M=\varnothing$, then

$A(\sigma) \backslash M=\{x \in X$ : there exists a bounded global solution of $\tilde{\varphi}$ at $\sigma$ through $x\}$.

\section{Collective tube conditions for INDS}

The tube conditions are essential to assure that a continuous flow and its associated impulsive flow have controled behavior in their evolutions near the impulsive set. In [4], the authors present the definition of collective tube conditions in order to establish an upper semicontinuity result for global attractors of impulsive autonomous systems. Using their ideas, we introduce the notion of collective tube conditions for a family of impulsive nonautonomous dynamical systems.

Throughout this section, we shall consider for each $\eta \in[0,1]$, an $\operatorname{INDS}\left[\left(\varphi_{\eta}, \theta\right)_{(X, \Sigma)}, M_{\eta}, I_{\eta}\right]$ with a $\left(\tilde{\varphi}_{\eta}, \mathfrak{D}\right)$-impulsive cocycle attractor $\hat{A}_{\eta}$ satisfying the following conditions:

(H1) $\varphi_{\eta}(t, \sigma) x \stackrel{\eta \rightarrow 0^{+}}{\longrightarrow} \varphi_{0}(t, \sigma) x$ uniformly in bounded subsets of $\mathbb{R}_{+} \times \Sigma \times X$.

(H2) $\mathrm{d}_{H}\left(M_{\eta}, M_{0}\right)+\mathrm{d}_{H}\left(M_{0}, M_{\eta}\right) \stackrel{\eta \rightarrow 0^{+}}{\longrightarrow} 0$.

(H3) Given $\epsilon>0$ and $w_{0} \in M_{0}$ there exists $\delta>0$ such that if $\eta \in[0, \delta), w \in M_{\eta}$ and $\mathrm{d}\left(w, w_{0}\right)<\delta$ then $\mathrm{d}\left(I_{\eta}(w), I_{0}\left(w_{0}\right)\right)<\epsilon$.

(H4) $I_{\eta}\left(M_{\eta}\right) \bigcap M_{\eta}=\varnothing$ for all $\eta \in[0,1]$.

According to Remark 2.5, we may consider for each $\eta \in[0,1]$, the impulsive skew-product semiflow associated with $\left[\left(\varphi_{\eta}, \theta\right)_{(X, \Sigma)}, M_{\eta}, I_{\eta}\right]$, given by $\left\{\left(\mathbb{X}, \Pi_{\eta}, \mathbb{M}_{\eta}, \mathbb{I}_{\eta}\right)\right\}_{\eta \in[0,1]}$. Inspired by 4 , Definition 3.3], we present the following.

Definition 3.1. We say that a point $\left(w_{0}, \sigma_{0}\right) \in \mathbb{M}_{0}$ satisfies the collective strong tube condition (C-STC) if given:

- $\left\{\eta_{k}\right\} \subset[0,1]$ such that $\eta_{k} \stackrel{k \rightarrow \infty}{\longrightarrow} 0$;

- $w_{\eta_{k}} \in M_{\eta_{k}}, k \geqslant 1$, with $w_{\eta_{k}} \stackrel{k \rightarrow \infty}{\longrightarrow} w_{0}$;

- $\left\{\sigma_{\eta_{k}}\right\} \subset \Sigma$ with $\sigma_{\eta_{k}} \stackrel{k \rightarrow \infty}{\longrightarrow} \sigma_{0}$,

there exists $\lambda_{0}>0$ such that for each $0<\lambda \leqslant \lambda_{0}$ one can find $\delta=\delta(\lambda)>0$ such that for each $j \in\{0\} \bigcup\left\{\eta_{k}\right\}$ we have a $\lambda$-tube $\mathbb{F}_{j}\left(\mathbb{L}_{j},[0,2 \lambda]\right)$ through $\left(w_{j}, \sigma_{j}\right)$ with section $\mathbb{S}_{j}=$ $\mathbb{F}_{j}\left(\mathbb{L}_{j},[0,2 \lambda]\right) \bigcap \mathbb{M}_{j}$ and $B\left(\left(w_{j}, \sigma_{j}\right), \delta\right) \subset \mathbb{F}_{j}\left(\mathbb{L}_{j},[0,2 \lambda]\right)$.

Moreover, if $\mathbb{F}_{j}\left(\mathbb{L}_{j},[0, \lambda]\right) \bigcap \mathbb{I}_{j}\left(\mathbb{M}_{j}\right)=\varnothing$ for all $j$ then we say that $\left(w_{0}, \sigma_{0}\right)$ satisfies the collective special strong tube condition (C-SSTC). 
The reader may consult [4] for examples and more details of collective tube conditions on autonomous impulsive systems. In the case of impulsive nonautonomous systems, we present the following definition for the tube conditions.

Definition 3.2. Let $\left\{\left[\left(\varphi_{\eta}, \theta\right)_{(X, \Sigma)}, M_{\eta}, I_{\eta}\right]\right\}_{\eta \in[0,1]}$ be a family of INDS's. We say that a point $w_{0} \in M_{0}$ satisfies the $\varphi$-collective strong tube condition ( $\left.\varphi-\mathbf{C}-\mathbf{S T C}\right)$ if for each $\sigma \in \Sigma$, the pair $\left(w_{0}, \sigma\right)$ satisfies C-STC with respect to the family of impulsive skew-product $\left\{\left(\mathbb{X}, \Pi_{\eta}, \mathbb{M}_{\eta}, \mathbb{I}_{\eta}\right)\right\}_{\eta \in[0,1]}$. Also, $w_{0} \in M_{0}$ satisfies the $\varphi$-collective special strong tube condition $\left(\varphi-\right.$ C-SSTC) if for each $\sigma \in \Sigma$, the pair $\left(w_{0}, \sigma\right)$ satisfies C-SSTC with respect to the family of impulsive skew-product $\left\{\left(\mathbb{X}, \Pi_{\eta}, \mathbb{M}_{\eta}, \mathbb{I}_{\eta}\right)\right\}_{\eta \in[0,1]}$.

Example 3.3 (Collective tube conditions). Let $C_{b}(\mathbb{R})$ be the Banach space of continuous and bounded functions from $\mathbb{R}$ to $\mathbb{R}$ with the supremum norm, and $f \in C_{b}(\mathbb{R})$ be a function such that for each $\alpha>0$ there exists $c_{\alpha}>0$ satisfying

$$
c_{\alpha} \leqslant \int_{x}^{\alpha+x} f(u) d u \quad \text { for all } x \in \mathbb{R} .
$$

Consider for each $\tau \in \mathbb{R}$ the translation $f_{\tau}(\cdot)=f(\tau+\cdot)$, and assume that $f$ is such that $\Sigma=\overline{\left\{f_{\tau}: \tau \in \mathbb{R}\right\}}$ is compact in $C_{b}(\mathbb{R})$ (we could consider $f$ globally Lipschitz or almost periodic, for instance, and use Proposition 2.1 from [13]). For each $t \in \mathbb{R}$, consider the group $\theta_{t}: \Sigma \rightarrow \Sigma$ given by $\theta_{t} g=g_{t}$. Now, for each $\eta \in[0,1]$, consider the $\operatorname{INDS}\left[\left(\varphi_{\eta}, \theta\right)_{(\mathbb{R}, \Sigma)}, M_{\eta}, I_{\eta}\right]$ given by

$$
\varphi_{\eta}(t, g) x_{0}=x_{0} \exp \left(-\int_{0}^{t}[g(u)+\eta] d u\right) \text { for all }\left(t, g, x_{0}\right) \in \mathbb{R}_{+} \times \Sigma \times \mathbb{R},
$$

$M_{\eta}=\{-1-\eta, 1+\eta\}, I_{\eta}(-1-\eta)=-2-\eta$ and $I_{\eta}(1+\eta)=2+\eta$. Note that condition (3.1) ensures 2.1) of Definition 2.2.

This INDS is generated by the family of initial value ordinary differential equations given by

$$
\left\{\begin{array}{l}
\dot{x}=[f(t)+\eta] x, \quad t \geqslant 0, \\
x(0)=x_{0} \in \mathbb{R}
\end{array}\right.
$$

for each $\eta \in[0,1]$.

Note that conditions (H1)-(H4) are satisfied, and we claim that both points in $M_{0}$ satisfies $\varphi$-C-SSTC. We will prove it for $w_{0}=1$, and the other is analogous.

In fact, let $\eta_{k} \stackrel{k \rightarrow \infty}{\longrightarrow} 0^{+}$with $\eta_{k}<\frac{1}{2}$ for all $k \geqslant 1, w_{k}=1+\eta_{k}$ and consider $\left\{\left(\mathbb{X}, \Pi_{\eta}, \mathbb{M}_{\eta}, \mathbb{I}_{\eta}\right)\right\}_{\eta \in[0,1]}$ the family of impulsive skew-product semiflow associated with the family of $\operatorname{INDS}\left\{\left[\left(\varphi_{\eta}, \theta\right)_{(\mathbb{R}, \Sigma)}\right.\right.$, $\left.\left.M_{\eta}, I_{\eta}\right]\right\}_{\eta \in[0,1]}$.

Since $f$ is bounded, if $M=\sup _{u \in \mathbb{R}} f(u)$ then $\int_{x}^{\alpha+x} f(u) d u \leqslant M \alpha$ for all $x \in \mathbb{R}$ and $\alpha>0$. Therefore, we can choose $\lambda_{0}>0$ such that $e^{(M+1) \lambda}<\frac{4}{3}$ for all $0<\lambda \leqslant \lambda_{0}$. Now, let $0<\lambda \leqslant \lambda_{0}$ 
and consider the set $\mathbb{L}_{k}=\bigcup_{g \in \Sigma} \Pi_{\eta_{k}}(\lambda)\left(w_{k}, g\right)$ for each $k \geqslant 0$, where $\eta_{0}=0$, and note that

$$
(x, h) \in \mathbb{F}_{\eta_{k}}\left(\mathbb{L}_{k},[0,2 \lambda]\right) \quad \text { iff } \quad x=w_{k} \exp \left(\int_{0}^{s}\left[h(u)+\eta_{k}\right] d u\right) \text {, for some } s \in[-\lambda, \lambda] .
$$

With $c_{\lambda}>0$ given in 3.1 , choose $\delta=\delta(\lambda)$ such that

$$
0<\delta<\min \left\{1-e^{-c_{\lambda}}, e^{c_{\lambda}}-1\right\}
$$

Consider $x \in \mathbb{R}$ such that $x \in\left(w_{k}-\delta, w_{k}+\delta\right)$ and $h \in \Sigma$. Define the function $\beta(s)=$ $\int_{0}^{s}\left[h(u)+\eta_{k}\right] d u$ for $-\lambda \leqslant s \leqslant \lambda$. Note that 3.1 holds for $h$ and it implies that $h(u) \geqslant 0$ for all $u \in \mathbb{R}$, consequently

$$
\beta(-\lambda) \leqslant \beta(s) \leqslant \beta(\lambda) \text { for all }-\lambda \leqslant s \leqslant \lambda .
$$

Moreover $\beta(-\lambda) \leqslant-c_{\lambda}$ and $\beta(\lambda) \geqslant c_{\lambda}$, and since $\beta$ is continuous we have $\beta([-\lambda, \lambda]) \supset$ $\left[-c_{\lambda}, c_{\lambda}\right]$. From our choice of $\delta$ we have

$$
w_{k} e^{-c_{\lambda}}<x<w_{k} e^{c_{\lambda}}
$$

therefore $\ln \left(\frac{x}{w_{k}}\right) \in\left[-c_{\lambda}, c_{\lambda}\right]$. Thus there exists $-\lambda \leqslant t \leqslant \lambda$ such that $\beta(t)=\ln \left(\frac{x}{w_{k}}\right)$, which implies

$$
x=w_{k} \exp \left(\int_{0}^{t}\left[h(u)+\eta_{k}\right] d u\right),
$$

and shows that $(x, h) \in \mathbb{F}_{\eta_{k}}\left(\mathbb{L}_{k},[0,2 \lambda]\right)$.

By our choice of $\lambda_{0}$ we have $\mathbb{F}_{\eta_{k}}\left(\mathbb{L}_{k},[0, \lambda]\right) \bigcap I_{\eta_{k}}\left(\mathbb{M}_{\eta_{k}}\right)=\varnothing$ for each $k \geqslant 1$. Therefore, $w_{0} \in M_{0}$ satisfies $\varphi$-C-SSTC.

Condition (H2) provides us a simple and useful result.

Lemma 3.4. If $\left\{\eta_{k}\right\} \subset[0,1]$ and $\left\{w_{k}\right\} \subset X$ are sequences such that $\eta_{k} \stackrel{k \rightarrow \infty}{\longrightarrow} 0, w_{k} \in M_{\eta_{k}}$ for $k \geqslant 1$ and $w_{k} \stackrel{k \rightarrow \infty}{\longrightarrow} w_{0}$, then $w_{0} \in M_{0}$.

Proof. From (H2) we have

$$
\mathrm{d}_{H}\left(w_{0}, M_{0}\right)=\inf _{z \in M_{0}} d\left(w_{0}, z\right) \leqslant \inf _{z \in M_{0}}\left(d\left(w_{0}, w_{k}\right)+d\left(w_{k}, z\right)\right) \leqslant d\left(w_{0}, w_{k}\right)+\mathrm{d}_{H}\left(M_{\eta_{k}}, M_{0}\right) \stackrel{k \rightarrow \infty}{\longrightarrow} 0,
$$

that is, $w_{0} \in M_{0}$, since $M_{0}$ is closed.

q.e.d.

From Definition 3.2 we have directly the following result.

Lemma 3.5. Assume that $w_{0} \in M_{0}$ satisfies $\varphi$-C-STC. If a sequence $\left\{\eta_{k}\right\} \subset[0,1]$ is such that $\eta_{k} \stackrel{k \rightarrow \infty}{\longrightarrow} 0, w_{k} \in M_{\eta_{k}}, k \geqslant 1$, is a sequence of points with $w_{k} \stackrel{k \rightarrow \infty}{\longrightarrow} w_{0}$, and $\left\{\sigma_{k}\right\}$ is a sequence in $\Sigma$ with $\sigma_{k} \stackrel{k \rightarrow \infty}{\longrightarrow} \sigma_{0}$ then there exists an integer $k_{1} \geqslant k_{0}$ such that $B\left(\left(w_{0}, \sigma_{0}\right), \frac{\delta}{2}\right) \subset B\left(\left(w_{k}, \sigma_{k}\right), \delta\right) \subset$ $\mathbb{F}_{\eta_{k}}\left(\mathbb{L}_{k},[0,2 \lambda]\right)$ for all $k \geqslant k_{1}$. 
Lemma 3.6. Assume that every point of $M_{0}$ satisfies $\varphi$-C-STC. Let $x_{0} \in X \backslash M_{0}, \sigma_{0} \in \Sigma$, $\left\{x_{k}\right\} \subset X$ and $\left\{\sigma_{k}\right\} \subset \Sigma$ be sequences such that $x_{k} \stackrel{k \rightarrow \infty}{\longrightarrow} x_{0}$ and $\sigma_{k} \stackrel{k \rightarrow \infty}{\longrightarrow} \sigma_{0}$. If $\left\{\eta_{k}\right\} \subset[0,1]$ is a sequence such that $\eta_{k} \stackrel{k \rightarrow \infty}{\longrightarrow} 0$, then

$$
\lim _{k \rightarrow \infty} \phi_{\eta_{k}}\left(x_{k}, \sigma_{k}\right)=\phi_{0}\left(x_{0}, \sigma_{0}\right),
$$

where $\phi_{\eta_{k}}\left(x_{k}, \sigma_{k}\right)$ represents the smallest positive time such that the positive semitrajectory of $x_{k}$ at the fiber $\sigma_{k}$ meets $M_{\eta_{k}}$.

Proof. Since each point in $M_{0} \times \Sigma$ satisfies C-STC and we have condition (H1), the result follows by [4, Theorem 3.12].

Proposition 3.7. Let $\left\{\left[\left(\varphi_{\eta}, \theta\right)_{(X, \Sigma)}, M_{\eta}, I_{\eta}\right]\right\}_{\eta \in[0,1]}$ be a family of INDS, $x_{0} \in X \backslash M_{0}, \sigma_{0} \in \Sigma$, $\left\{x_{k}\right\} \subset X,\left\{\sigma_{k}\right\} \subset \Sigma$ and $\left\{\eta_{k}\right\} \subset[0,1]$ be sequences such that $x_{k} \stackrel{k \rightarrow \infty}{\longrightarrow} x_{0}, \sigma_{k} \stackrel{k \rightarrow \infty}{\longrightarrow} \sigma_{0}$ and $\eta_{k} \stackrel{k \rightarrow \infty}{\longrightarrow} 0$. Assume that each point of $M_{0}$ satisfies $\varphi-C-S T C$. Given $t \geqslant 0$, there exists a sequence $\left\{\epsilon_{k}\right\} \subset[0, \infty)$ such that $\epsilon_{k} \stackrel{k \rightarrow \infty}{\longrightarrow} 0$ and

$$
\tilde{\varphi}_{\eta_{k}}\left(t+\epsilon_{k}, \sigma_{k}\right) x_{k} \stackrel{k \rightarrow \infty}{\longrightarrow} \tilde{\varphi}_{0}(t, \sigma) x_{0}
$$

Proof. If follows by [4, Corollary 3.17] using the fact that each point from $M_{0} \times \Sigma$ satisfies C-STC. q.e.d.

Proposition 3.8. Let $\left\{\left[\left(\varphi_{\eta}, \theta\right)_{(X, \Sigma)}, M_{\eta}, I_{\eta}\right]\right\}_{\eta \in[0,1]}$ be a family of INDS, $x_{0} \in X \backslash M_{0}, \sigma_{0} \in \Sigma$, $\left\{x_{k}\right\} \subset X,\left\{\sigma_{k}\right\} \subset \Sigma$ and $\left\{\eta_{k}\right\} \subset[0,1]$ be sequences such that $x_{k} \stackrel{k \rightarrow \infty}{\longrightarrow} x_{0}, \sigma_{k} \stackrel{k \rightarrow \infty}{\longrightarrow} \sigma_{0}$ and $\eta_{k} \stackrel{k \rightarrow \infty}{\longrightarrow} 0$. Assume that each point of $M_{0}$ satisfies $\varphi-C-S T C$. If $t \geqslant 0$ is not an impulsive time of $\tilde{\varphi}(\cdot, \sigma) x$ (see Definition 2.4), then

$$
\tilde{\varphi}_{\eta_{k}}\left(t, \sigma_{k}\right) x_{k} \stackrel{k \rightarrow \infty}{\longrightarrow} \tilde{\varphi}_{0}(t, \sigma) x_{0}
$$

Proof. If follows by [4, Proposition 3.14, Remark 3.15] using the fact that each point from $M_{0} \times \Sigma$ satisfies C-STC for $\Pi$.

q.e.d.

Proposition 3.9. Let $x_{0} \in X \backslash M_{0}, \sigma_{0} \in \Sigma,\left\{x_{k}\right\} \subset X,\left\{\sigma_{k}\right\} \subset \Sigma$ and $\left\{\eta_{k}\right\} \subset[0,1]$ be sequences such that $x_{k} \stackrel{k \rightarrow \infty}{\longrightarrow} x_{0}, \sigma_{k} \stackrel{k \rightarrow \infty}{\longrightarrow} \sigma_{0}$ and $\eta_{k} \stackrel{k \rightarrow \infty}{\longrightarrow} 0$. Assume that each point of $M_{0}$ satisfies $\varphi$-C-STC. Then, given $\left\{\alpha_{k}\right\} \subset[0,1]$ such that $\alpha_{k} \stackrel{k \rightarrow \infty}{\longrightarrow} 0$ we have $\tilde{\varphi}_{\eta_{k}}\left(\alpha_{k}, \sigma_{k}\right) x_{k} \stackrel{k \rightarrow \infty}{\longrightarrow} x_{0}$.

Proof. Since $x_{0} \notin M_{0}$, it follows from Lemma 3.6 that $\phi_{\eta_{k}}\left(x_{k}, \sigma_{k}\right) \stackrel{k \rightarrow \infty}{\longrightarrow} \phi_{0}\left(x_{0}, \sigma_{0}\right)$. Then there exists $\bar{k} \in \mathbb{N}$ such that $\alpha_{k}<\phi_{\eta_{k}}\left(x_{k}, \sigma_{k}\right)$ for all $k \geqslant \bar{k}$, and by (H1) we have

$$
\tilde{\varphi}_{\eta_{k}}\left(\alpha_{k}, \sigma_{k}\right) x_{k}=\varphi_{\eta_{k}}\left(\alpha_{k}, \sigma_{k}\right) x_{k} \stackrel{k \rightarrow \infty}{\longrightarrow} \varphi_{0}\left(0, \sigma_{0}\right) x_{0}=x_{0}
$$

which concludes the proof.

q.e.d. 
Next, to end this section, we present two new results that will give us one of the necessary conditions to ensure the upper semicontinuity of impulsive cocycle attractors (see condition (b) of Theorem 4.4.

Lemma 3.10. Let $\mathfrak{D}$ be a universe and $\left[(\varphi, \theta)_{(X, \Sigma)}, M, I\right]$ be an INDS with $(\tilde{\varphi}, \mathfrak{D})$-impulsive cocycle attractor $\hat{A}$ such that $I(M) \bigcap M=\varnothing$. Let $\hat{K} \in \mathfrak{D}$ be a pullback $\mathfrak{D}$-absorbing set such that $A(\sigma) \subset K(\sigma)$ for all $\sigma \in \Sigma$. Then $\hat{A}$ is uniquely determined by

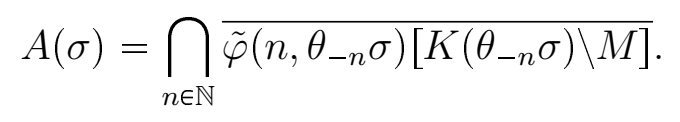

Proof. By the pullback $(\tilde{\varphi}, \mathfrak{D})$-attracting property of $\hat{A}$, since $\hat{K} \in \mathfrak{D}$, we have

$$
\bigcap_{n \in \mathbb{N}} \overline{\tilde{\varphi}\left(n, \theta_{-n} \sigma\right)\left[K\left(\theta_{-n} \sigma\right) \backslash M\right]} \subset \overline{\bigcap_{n \in \mathbb{N}} \tilde{\varphi}\left(n, \theta_{-n} \sigma\right) K\left(\theta_{-n} \sigma\right)} \subset A(\sigma) \quad \text { for all } \sigma \in \Sigma \text {. }
$$

On the other hand, let $n \geqslant 1, \sigma \in \Sigma$ and $\hat{D} \in \mathfrak{D}$. There exists $t_{0}=t_{0}\left(\theta_{-n} \sigma, \hat{D}\right)>0$ such that

$$
\tilde{\varphi}\left(t, \theta_{-t-n} \sigma\right) D\left(\theta_{-t-n} \sigma\right) \subset K\left(\theta_{-n} \sigma\right) \text { for all } t \geqslant t_{0}\left(\theta_{-n} \sigma, \hat{D}\right) .
$$

Since $I(M) \bigcap M=\varnothing$, we have

$$
\tilde{\varphi}\left(t, \theta_{-t-n} \sigma\right) D\left(\theta_{-t-n} \sigma\right) \subset K\left(\theta_{-n} \sigma\right) \backslash M \quad \text { for all } t \geqslant t_{0}\left(\theta_{-n} \sigma, \hat{D}\right) .
$$

Consequently,

$$
\tilde{\varphi}\left(n, \theta_{-n} \sigma\right) \tilde{\varphi}\left(t, \theta_{-t-n} \sigma\right) D\left(\theta_{-t-n} \sigma\right) \subset \tilde{\varphi}\left(n, \theta_{-n} \sigma\right)\left[K\left(\theta_{-n} \sigma\right) \backslash M\right] \quad \text { for all } \quad t \geqslant t_{0}\left(\theta_{-n} \sigma, \hat{D}\right),
$$

that is,

$$
\tilde{\varphi}\left(n+t, \theta_{-t-n} \sigma\right) D\left(\theta_{-t-n} \sigma\right) \subset \tilde{\varphi}\left(n, \theta_{-n} \sigma\right)\left[K\left(\theta_{-n} \sigma\right) \backslash M\right] \text { for all } t \geqslant t_{0}\left(\theta_{-n} \sigma, \hat{D}\right) .
$$

Thus, we may conclude that

$$
\tilde{\varphi}\left(s, \theta_{-s} \sigma\right) D\left(\theta_{-s} \sigma\right) \subset \overline{\tilde{\varphi}\left(n, \theta_{-n} \sigma\right)\left[K\left(\theta_{-n} \sigma\right) \backslash M\right]} \text { for all } s \geqslant t_{0}\left(\theta_{-n} \sigma, \hat{D}\right)+n .
$$

By (3.3), for each fixed $n \in \mathbb{N}$, the nonautonomous set $\left\{\overline{\tilde{\varphi}\left(n, \theta_{-n} \sigma\right)\left[K\left(\theta_{-n} \sigma\right) \backslash M\right]}\right\}_{\sigma \in \Sigma}$ is closed and pullback $(\tilde{\varphi}, \mathfrak{D})$-attracting. By minimality of the cocycle attractor $\hat{A}$, we have

$$
A(\sigma) \subset \overline{\tilde{\varphi}\left(n, \theta_{-n} \sigma\right)\left[K\left(\theta_{-n} \sigma\right) \backslash M\right]}
$$

for all $\sigma \in \Sigma$. Since $n$ is taken arbitrary, we have $A(\sigma) \subset \bigcap_{n \in \mathbb{N}} \overline{\tilde{\varphi}\left(n, \theta_{-n} \sigma\right)\left[K\left(\theta_{-n} \sigma\right) \backslash M\right]}$, for each $\sigma \in \Sigma$.

q.e.d.

Theorem 3.11. Let $\mathfrak{D}$ be a universe and $\left[(\varphi, \theta)_{(X, \Sigma)}, M, I\right]$ be an $\operatorname{INDS}$ with $(\tilde{\varphi}, \mathfrak{D})$-impulsive cocycle attractor $\hat{A}$ such that $I(M) \bigcap M=\varnothing$. Let $\hat{K} \in \mathfrak{D}$ be a closed pullback $\mathfrak{D}$-absorbing such that $A(\sigma) \subset K(\sigma)$ for all $\sigma \in \Sigma$ and $\overline{\bigcup_{\sigma \in \Sigma} K(\sigma)}$ be compact. Assume that for each $\sigma \in \Sigma$ :

(a) $\lim _{s \rightarrow 0^{+}} \mathrm{d}_{H}\left(K\left(\theta_{-s} \sigma\right), K(\sigma)\right)=0$; 
(b) For $x \in M \bigcap K(\sigma)$ and $t>0$, there exists a sequence $\left\{z_{k}\right\} \subset K\left(\theta_{-t} \sigma\right) \backslash M$ such that $\tilde{\varphi}\left(t, \theta_{-t} \sigma\right) z_{k} \stackrel{k \rightarrow \infty}{\longrightarrow} x$.

Then $\lim _{s \rightarrow 0^{+}} \mathrm{d}_{H}\left(A\left(\theta_{-s} \sigma\right), A(\sigma)\right)=0$ for each $\sigma \in \Sigma$.

Proof. Suppose to the contrary there exist $\sigma \in \Sigma, \delta>0$, a sequence $s_{k} \stackrel{k \rightarrow \infty}{\longrightarrow} 0^{+}$and a sequence $\left\{x_{k}\right\}_{x \geqslant 1}$ such that $x_{k} \in A\left(\theta_{-s_{k}} \sigma\right)$ and $d\left(x_{k}, A(\sigma)\right) \geqslant \delta$ for all $k \geqslant 1$. By the compactness of $\overline{\bigcup_{\sigma \in \Sigma} K(\sigma)}$ we may assume $x_{k} \stackrel{k \rightarrow \infty}{\longrightarrow} x_{0}$. By hypothesis $A\left(\theta_{-s_{k}} \sigma\right) \subset K\left(\theta_{-s_{k}} \sigma\right)$ for all $k \geqslant 1$, thus condition (a) implies in $x_{0} \in K(\sigma)$.

If $x_{0} \notin M$ then we may assume that $x_{k} \notin M$ for all $k \geqslant 1$. Using Proposition 3.9 we obtain $\tilde{\varphi}\left(s_{k}, \theta_{-s_{k}} \sigma\right) x_{k} \stackrel{k \rightarrow \infty}{\longrightarrow} x_{0}$, and by the invariance of the nonautonomous set $\hat{A} \backslash M$ we have $\tilde{\varphi}\left(s_{k}, \theta_{-s_{k}} \sigma\right) x_{k} \in A(\sigma)$ for all $k \geqslant 1$. Consequently, $x_{0} \in A(\sigma)$ which is a contradiction.

If $x_{0} \in M$ then, for each fixed $n \in \mathbb{N}$, using condition (b), there is a sequence $\left\{z_{k}^{n}\right\} \subset$ $K\left(\theta_{-n} \sigma\right) \backslash M$ such that

$$
\tilde{\varphi}\left(n, \theta_{-n} \sigma\right) z_{k}^{n} \stackrel{k \rightarrow \infty}{\longrightarrow} x_{0}
$$

Hence, $x_{0} \in \overline{\tilde{\varphi}\left(n, \theta_{-n} \sigma\right)\left[K\left(\theta_{-n} \sigma\right) \backslash M\right]}$ for all $n \in \mathbb{N}$. By Lemma 3.10, $x_{0} \in A(\sigma)$ which is a contradiction.

q.e.d.

This result is most useful in the form of the next corollary.

Corollary 3.12. Let $\mathfrak{D}$ be a universe, $\left[(\varphi, \theta)_{(X, \Sigma)}, M, I\right]$ be an INDS such that $I(M) \bigcap M=$ $\varnothing$ with $(\tilde{\varphi}, \mathfrak{D})$-impulsive cocycle attractor $\hat{A}$ and $K_{0} \subset X$ be a compact set. Assume that $\hat{K} \in \mathfrak{D}, K(\sigma)=K_{0}$ for every $\sigma \in \Sigma$, is a closed pullback $\mathfrak{D}$-absorbing such that $A(\sigma) \subset K_{0}$ for all $\sigma \in \Sigma$. If for each $x \in M \bigcap K_{0}$ and $t>0$, there exists a sequence $\left\{z_{k}\right\} \subset K_{0} \backslash M$ such that $\tilde{\varphi}\left(t, \theta_{-t} \sigma\right) z_{k} \stackrel{k \rightarrow \infty}{\longrightarrow} x$, then $\lim _{k \rightarrow \infty} \mathrm{d}_{H}\left(A\left(\theta_{-1 / k} \sigma\right), A(\sigma)\right)=0$ for each $\sigma \in \Sigma$.

\section{Upper semicontinuity of impulsive cocycle attractors}

Here we deal with the upper semicontinuity at 0 for a family of $\left(\tilde{\varphi}_{\eta}, \mathfrak{D}\right)$-impulsive cocycle attractors $\left\{\hat{A}_{\eta}\right\}_{\eta \in[0,1]}$, that is, we will prove that for each $\sigma \in \Sigma$ we have

$$
\lim _{\eta \rightarrow 0^{+}} \mathrm{d}_{H}\left(A_{\eta}(\sigma), A_{0}(\sigma)\right)=0 .
$$

We recall that (4.1) is equivalent to the following statement: given sequences $\left\{\eta_{k}\right\} \subset[0,1]$ with $\eta_{k} \stackrel{k \rightarrow \infty}{\longrightarrow} 0$ and $x_{k} \in A_{\eta_{k}}(\sigma)$, there exists a subsequence $\left\{k_{j}\right\}_{j \geqslant 1}$ of the positive integers with $k_{j} \stackrel{j \rightarrow \infty}{\longrightarrow} \infty$ and a point $x_{0} \in A_{0}(\sigma)$ such that $x_{k_{j}} \stackrel{j \rightarrow \infty}{\longrightarrow} x_{0}$. 
To obtain this upper semicontinuity we consider and/or assume:

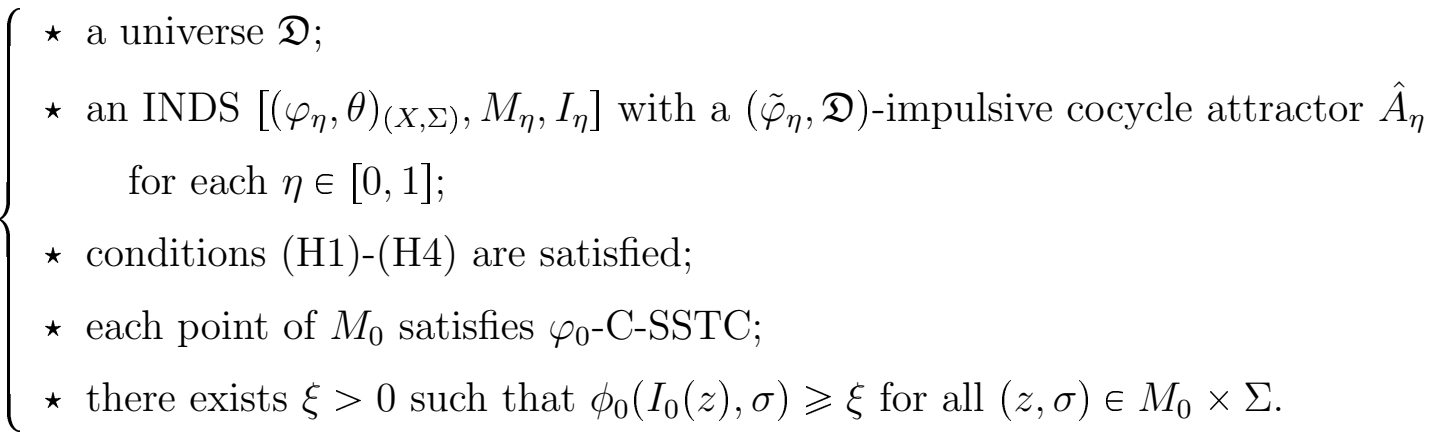

In what follows we present three auxiliary lemmas, which will help us to prove our main theorem. For the following results, we will additionally set:

Fix $\sigma \in \Sigma$ and let $\left\{\eta_{k}\right\},\left\{\epsilon_{k}\right\} \subset[0,1]$ be sequences such that $\eta_{k}, \epsilon_{k} \stackrel{k \rightarrow \infty}{\longrightarrow} 0$. Let $\sigma_{k}=\theta_{\epsilon_{k}} \sigma$ and $x_{k} \in A_{\eta_{k}}\left(\sigma_{k}\right) \backslash M_{\eta_{k}}$ for each $k \geqslant 1$ and $\psi_{k}$ be a global solution of $\tilde{\varphi}_{\eta_{k}}$ through $x_{k}$ at $\sigma_{k}$.

Lemma 4.1. Assume that (A1) and (A2) hold. Let $r \geqslant 0, y_{k}=\psi_{k}(-r)$ for each $k \geqslant 1$, and assume that $y_{k} \stackrel{k \rightarrow \infty}{\longrightarrow} y_{0} \in M_{0}$. Then $s_{k}=\phi_{\eta_{k}}\left(y_{k}, \theta_{-r} \sigma_{k}\right) \stackrel{k \rightarrow \infty}{\longrightarrow} 0$, and for each $t \geqslant 0$ there is a sequence $\left\{\beta_{k}\right\} \subset[0,1]$ with $\beta_{k} \stackrel{k \rightarrow \infty}{\longrightarrow} 0$ such that $\tilde{\varphi}_{\eta_{k}}\left(t+\beta_{k}, \theta_{-r} \sigma_{k}\right) y_{k} \stackrel{k \rightarrow \infty}{\longrightarrow} \varphi_{0}\left(t, \theta_{-r} \sigma\right) I_{0}\left(y_{0}\right)$. In particular, if $0<t<\xi$ then $\tilde{\varphi}_{\eta_{k}}\left(t, \theta_{-r} \sigma_{k}\right) y_{k} \stackrel{k \rightarrow \infty}{\longrightarrow} \varphi_{0}\left(t, \theta_{-r} \sigma\right) I_{0}\left(y_{0}\right)$.

Proof. Note that by (H2) there exists a subsequence of $\left\{\eta_{k}\right\}$, which we will denote the same, and a sequence $\left\{w_{k}\right\} \subset X$ with $w_{k} \in M_{\eta_{k}}$ such that $w_{k} \stackrel{k \rightarrow \infty}{\longrightarrow} y_{0}$. Since $M_{0}$ satisfies $\varphi_{0}$-C-SSTC it follows by Lemma 3.5 that there exist $\lambda, \delta>0$ and $k_{1} \in \mathbb{N}$ such that

$$
B\left(\left(w_{k}, \theta_{-r} \sigma_{k}\right), \delta\right) \subset \mathbb{F}_{\eta_{k}}\left(\mathbb{L}_{k},[0,2 \lambda]\right) \quad \text { for all } k \geqslant k_{1},
$$

where $\mathbb{F}_{\eta_{k}}\left(\mathbb{L}_{k},[0,2 \lambda]\right)$ is a $\lambda$-tube through $\left(w_{k}, \theta_{-r} \sigma_{k}\right)$ with section $\mathbb{S}_{k}=\mathbb{F}_{\eta_{k}}\left(\mathbb{L}_{k},[0,2 \lambda]\right) \bigcap \mathbb{M}_{\eta_{k}}$ and $\mathbb{F}_{0}\left(\mathbb{L}_{0},[0,2 \lambda]\right)$ is a $\lambda$-tube through $\left(y_{0}, \theta_{-r} \sigma\right)$ with section $\mathbb{S}_{0}=\mathbb{F}_{0}\left(\mathbb{L}_{0},[0,2 \lambda]\right) \bigcap \mathbb{M}_{0}$. Moreover, we have $\mathbb{F}_{\eta_{k}}\left(\mathbb{L}_{k},[0, \lambda]\right) \bigcap \mathbb{I}\left(\mathbb{M}_{\eta_{k}}\right)=\varnothing$ and

$$
B\left(\left(y_{0}, \theta_{-r} \sigma\right), \frac{\delta}{2}\right) \subset \mathbb{F}_{0}\left(\mathbb{L}_{0},[0,2 \lambda]\right) \bigcap B\left(\left(w_{k}, \theta_{-r} \sigma_{k}\right), \delta\right) \quad \text { for all } k \geqslant k_{1} .
$$

Hence, there is $k_{2} \geqslant k_{1}$ such that $\left(y_{k}, \theta_{-r} \sigma_{k}\right) \in B\left(\left(w_{k}, \theta_{-r} \sigma_{k}\right), \delta\right)$ for all $k \geqslant k_{2}$. Taking a positive integer $m$ such that $m r>\lambda$, we have

$$
\tilde{\Pi}_{\eta_{k}}(m r)\left(\psi_{k}(-(m+1) r), \theta_{-(m+1) r} \sigma_{k}\right)=\left(y_{k}, \theta_{-r} \sigma_{k}\right)
$$

which implies that $\left(y_{k}, \theta_{-r} \sigma_{k}\right)$ belongs to the trajectory of the global solution of the impulsive skew-product semiflow $\tilde{\Pi}_{\eta_{k}}$ associated to $\tilde{\varphi}_{\eta_{k}}$, for some time $m r>\lambda$. Therefore, Proposition 2.10 implies that $\left(y_{k}, \theta_{-r} \sigma_{k}\right) \in \mathbb{F}_{\eta_{k}}\left(\mathbb{L}_{k},(\lambda, 2 \lambda]\right)$ for $k \geqslant k_{2}$ and, consequently, there is $\alpha_{k} \in(0, \lambda]$ such that $\Pi_{\eta_{k}}\left(\alpha_{k}\right)\left(y_{k}, \theta_{-r} \sigma_{k}\right) \in \mathbb{M}_{\eta_{k}}$, that is, $\varphi_{\eta_{k}}\left(\alpha_{k}, \theta_{-r} \sigma_{k}\right) y_{k} \in M_{\eta_{k}}$ for $k \geqslant k_{2}$. We may 
assume that $\alpha_{k} \stackrel{k \rightarrow \infty}{\longrightarrow} \alpha_{0} \in[0, \lambda]$ and from (H1) and Lemma 3.4 we obtain $\varphi_{\eta_{k}}\left(\alpha_{k}, \theta_{-r} \sigma_{k}\right) y_{k} \stackrel{k \rightarrow \infty}{\longrightarrow}$ $\varphi_{0}\left(\alpha_{0}, \theta_{-r} \sigma\right) y_{0} \in M_{0}$.

Since $\varphi_{0}\left((0, \lambda], \theta_{-r} \sigma\right) y_{0} \bigcap M_{0}=\varnothing$, it follows that $\alpha_{0}=0$. Hence, as $0<\phi_{\eta_{k}}\left(y_{k}, \theta_{-r} \sigma_{k}\right) \leqslant \alpha_{k}$ for all $k \geqslant k_{2}$, we obtain $\phi_{\eta_{k}}\left(y_{k}, \theta_{-r} \sigma_{k}\right) \stackrel{k \rightarrow \infty}{\longrightarrow} 0$.

Now, let $t \geqslant 0$. If $s_{k}=\phi_{\eta_{k}}\left(y_{k}, \theta_{-r} \sigma_{k}\right)$, then

$$
\varphi_{\eta_{k}}\left(s_{k}, \theta_{-r} \sigma_{k}\right) y_{k} \in M_{\eta_{k}} \text { and } \varphi_{\eta_{k}}\left(s_{k}, \theta_{-r} \sigma_{k}\right) y_{k} \stackrel{k \rightarrow \infty}{\longrightarrow} y_{0} .
$$

Moreover,

$$
\tilde{\varphi}_{\eta_{k}}\left(s_{k}, \theta_{-r} \sigma_{k}\right) y_{k} \stackrel{k \rightarrow \infty}{\longrightarrow} I_{0}\left(y_{0}\right) \notin M_{0}
$$

as $I_{0}\left(M_{0}\right) \bigcap M_{0}=\varnothing$. By Proposition 3.7, there is a sequence $\left\{\mu_{k}\right\} \subset[0,1]$ with $\mu_{k} \stackrel{k \rightarrow \infty}{\longrightarrow} 0$ such that

$$
\tilde{\varphi}_{\eta_{k}}\left(t+\mu_{k}+s_{k}, \theta_{-r} \sigma_{k}\right) y_{k}=\tilde{\varphi}_{\eta_{k}}\left(t+\mu_{k}, \theta_{s_{k}-r} \sigma_{k}\right) \tilde{\varphi}_{\eta_{k}}\left(s_{k}, \theta_{-r} \sigma_{k}\right) y_{k} \stackrel{k \rightarrow \infty}{\longrightarrow} \varphi_{0}\left(t, \theta_{-r} \sigma\right) I_{0}\left(y_{0}\right) .
$$

Now, it is enough to take $\beta_{k}=s_{k}+\mu_{k}$.

To end this proof note that for $0<t<\xi$, we have $t<\phi_{0}\left(I_{0}\left(y_{0}\right), \theta_{-r} \sigma\right)$ (see the last condition of $(\mathrm{A} 1))$. Since $\tilde{\varphi}_{\eta_{k}}\left(s_{k}, \theta_{-r} \sigma_{k}\right) y_{k} \stackrel{k \rightarrow \infty}{\longrightarrow} I_{0}\left(y_{0}\right)$ and $s_{k} \stackrel{k \rightarrow \infty}{\longrightarrow} 0, s_{k}<t<\phi_{\eta_{k}}\left(\tilde{\varphi}_{\eta_{k}}\left(s_{k}, \theta_{-r} \sigma_{k}\right) y_{k}, \theta_{s_{k}-r} \sigma_{k}\right)$ for $k$ sufficiently large, see Lemma 3.6 . Then

$$
\begin{aligned}
\tilde{\varphi}_{\eta_{k}}\left(t, \theta_{-r} \sigma_{k}\right) y_{k} & =\tilde{\varphi}_{\eta_{k}}\left(t-s_{k}, \theta_{s_{k}-r} \sigma_{k}\right) \tilde{\varphi}_{\eta_{k}}\left(s_{k}, \theta_{-r} \sigma_{k}\right) y_{k} \\
& =\varphi_{\eta_{k}}\left(t-s_{k}, \theta_{s_{k}-r} \sigma_{k}\right) \tilde{\varphi}_{\eta_{k}}\left(s_{k}, \theta_{-r} \sigma_{k}\right) y_{k} \stackrel{k \rightarrow \infty}{\longrightarrow} \varphi_{0}\left(t, \theta_{-r} \sigma\right) I_{0}\left(y_{0}\right) .
\end{aligned}
$$

q.e.d.

Lemma 4.2. Assume that (A1)-(A2) hold and assume additionally that there exists $r \geqslant 0$ such that $\psi_{k}(-r) \stackrel{k \rightarrow \infty}{\longrightarrow} x_{0} \in M_{0}$. If

$$
\overline{\bigcup_{k \geqslant 1} \psi_{k}(\mathbb{R})} \text { is compact, }
$$

then there exists a positive integer $m_{0}$ such that, for $m \geqslant m_{0}$, one can find $k_{0}^{m} \in \mathbb{N}$ such that

$$
\phi_{\eta_{k}}\left(\psi_{k}(-1 / m-r), \theta_{-1 / m-r} \sigma_{k}\right)>\frac{1}{m} \quad \text { for all } k \geqslant k_{0}^{m} .
$$

Proof. Since $\psi_{k}(-r) \stackrel{k \rightarrow \infty}{\longrightarrow} x_{0} \in M_{0}$, Lemma 4.1 implies that $t_{k}=\phi_{\eta_{k}}\left(\psi_{k}(-r), \theta_{-r} \sigma_{k}\right) \stackrel{k \rightarrow \infty}{\longrightarrow} 0$. Thus, we may assume that $0<t_{k}<\frac{\xi}{4}$ for all $k \geqslant 1$.

By (2.1), there exists $\epsilon_{x_{0}, \sigma}>0$ such that $\bigcup_{t \in\left(0, \epsilon_{x_{0}, \sigma}\right)}\left(F_{\varphi_{0}}\left(x_{0}, t, \theta_{-t} \sigma\right) \bigcap M_{0}\right)=\varnothing$. Choose $m_{0} \in \mathbb{N}$ such that $\frac{1}{m_{0}}<\min \left\{\epsilon_{x_{0}, \sigma}, \frac{\xi}{4}\right\}$ and fix $m \geqslant m_{0}$. Using (4.2), taking a subsequence if necessary, we may assume that $\psi_{k}(-1 / m-r) \stackrel{k \rightarrow \infty}{\longrightarrow} y_{m}$. Set $s_{k}=\phi_{\eta_{k}}\left(\psi_{k}(-1 / m-r), \theta_{-1 / m-r} \sigma_{k}\right)$ and suppose, arguing by contradiction, that there exists a subsequence of $\left\{s_{k}\right\}$, still denoted the same, such that

$$
s_{k} \leqslant \frac{1}{m} \quad \text { for all } \quad k \geqslant 1 \text {. }
$$


Hence, $\left\{s_{k}\right\}$ is bounded, and we can assume, without loss of generality, that $s_{k} \stackrel{k \rightarrow \infty}{\longrightarrow} \bar{s}$. Taking $z_{k}=\varphi_{\eta_{k}}\left(s_{k}, \theta_{-1 / m-r} \sigma_{k}\right) \psi_{k}\left(-\frac{1}{m}-r\right) \in M_{\eta_{k}}$ and using (H1), we obtain

$$
z_{k} \stackrel{k \rightarrow \infty}{\longrightarrow} \varphi_{0}\left(\bar{s}, \theta_{-1 / m-r} \sigma\right) y_{m}=w_{m} \in M_{0}
$$

Then by (H3) we have

$$
I_{\eta_{k}}\left(z_{k}\right)=\tilde{\varphi}_{\eta_{k}}\left(s_{k}, \theta_{-1 / m-r} \sigma_{k}\right) \psi_{k}(-1 / m-r) \stackrel{k \rightarrow \infty}{\longrightarrow} I_{0}\left(w_{m}\right) \in I_{0}\left(M_{0}\right) .
$$

Since $\phi_{0}\left(I_{0}\left(w_{m}\right), \theta_{\bar{s}-1 / m-r} \sigma\right) \geqslant \xi$ by the last condition of (A1), it follows by Lemma 3.6 that

$$
\phi_{\eta_{k}}\left(I_{\eta_{k}}\left(z_{k}\right), \theta_{s_{k}-1 / m-r} \sigma_{k}\right)>\frac{\xi}{2}>\frac{1}{m} \quad \text { for } k \text { sufficiently large. }
$$

Thus for such values of $k$ we have

$$
\begin{aligned}
\varphi_{\eta_{k}}\left(t_{k}+\frac{1}{m}-s_{k},\right. & \left.\theta_{s_{k}-1 / m-r} \sigma_{k}\right) I_{\eta_{k}}\left(z_{k}\right)=\varphi_{\eta_{k}}\left(t_{k}, \theta_{-r} \sigma_{k}\right) \varphi_{\eta_{k}}\left(\frac{1}{m}-s_{k}, \theta_{s_{k}-1 / m-r} \sigma_{k}\right) I_{\eta_{k}}\left(z_{k}\right) \\
& =\varphi_{\eta_{k}}\left(t_{k}, \theta_{-r} \sigma_{k}\right) \tilde{\varphi}_{\eta_{k}}\left(\frac{1}{m}-s_{k}, \theta_{s_{k}-1 / m-r} \sigma_{k}\right) I_{\eta_{k}}\left(z_{k}\right) \\
& =\varphi_{\eta_{k}}\left(t_{k}, \theta_{-r} \sigma_{k}\right) \tilde{\varphi}_{\eta_{k}}\left(\frac{1}{m}-s_{k}, \theta_{s_{k}-1 / m-r} \sigma_{k}\right) \tilde{\varphi}_{\eta_{k}}\left(s_{k}, \theta_{-1 / m-r} \sigma_{k}\right) \psi_{k}(-1 / m-r) \\
& =\varphi_{\eta_{k}}\left(t_{k}, \theta_{-r} \sigma_{k}\right) \tilde{\varphi}_{\eta_{k}}\left(\frac{1}{m}, \theta_{-1 / m-r} \sigma_{k}\right) \psi_{k}(-1 / m-r) \\
& =\varphi_{\eta_{k}}\left(t_{k}, \theta_{-r} \sigma_{k}\right) \psi_{k}(-r) \in M_{\eta_{k}},
\end{aligned}
$$

which is a contradiction, since

$$
t_{k}+\frac{1}{m}-s_{k}<t_{k}+\frac{1}{m}<\frac{\xi}{4}+\frac{1}{m_{0}}<\frac{\xi}{2}
$$

Therefore, there is $k_{0}^{m} \in \mathbb{N}$ such that $\phi_{\eta_{k}}\left(\psi_{k}(-1 / m-r), \theta_{-1 / m-r} \sigma_{k}\right)>\frac{1}{m}$ for all $k \geqslant k_{0}^{m}$. q.e.d.

Lemma 4.3. Assume that (A1)-(A2) and 4.2 hold, and $\psi_{k}(-r) \stackrel{k \rightarrow \infty}{\longrightarrow} x_{0} \in X$ for some $r \geqslant 0$. Then

(i) if $x_{0} \notin M_{0}$ then there exists a bounded global solution $\psi_{0}: \mathbb{R} \rightarrow X$ through $x_{0}$ at $\theta_{-r} \sigma$.

(ii) if $x_{0} \in M_{0}$ then there exists $m_{0} \in \mathbb{N}$ such that for $m \geqslant m_{0}$ the sequence $\left\{\psi_{k}(-1 / m-r)\right\}$ admits a subsequence with limit $u_{m} \notin M_{0}$ such that $u_{m} \stackrel{m \rightarrow \infty}{\longrightarrow} x_{0}$. Moreover, there is a bounded global solution $\psi_{0}^{m}$ of $\tilde{\varphi}_{0}$ through $u_{m}$ at $\theta_{-1 / m-r} \sigma$ for each $m \geqslant m_{0}$.

Proof. By 4.2), there exists a subsequence, which we denote the same, such that $\psi_{k}(-r-m) \stackrel{k \rightarrow \infty}{\longrightarrow} x_{-m}$ for all $m \geqslant 1$.

$\star$ Proof of $(i)$. Suppose firstly that $x_{-m} \notin M_{0}$ for all $m \geqslant 1$. By Proposition 3.7, for each $m \geqslant 1$, there exists a sequence $\left\{\mu_{k}^{m}\right\} \subset[0, \infty)$ such that $\mu_{k}^{m} \stackrel{k \rightarrow \infty}{\longrightarrow} 0$ and

$$
\tilde{\varphi}_{\eta_{k}}\left(1+\mu_{k}^{m}, \theta_{-r-m} \sigma_{k}\right) \psi_{k}(-r-m) \stackrel{k \rightarrow \infty}{\longrightarrow} \tilde{\varphi}_{0}\left(1, \theta_{-r-m} \sigma\right) x_{-m} .
$$

But $\tilde{\varphi}_{\eta_{k}}\left(1+\mu_{k}^{m}, \theta_{-r-m} \sigma_{k}\right) \psi_{k}(-r-m)=\tilde{\varphi}_{\eta_{k}}\left(\mu_{k}^{m}, \theta_{-(r+m-1)} \sigma_{k}\right) \psi_{k}(-r-m+1)$, and using Proposition 3.9 we obtain

$$
\tilde{\varphi}_{\eta_{k}}\left(1+\mu_{k}^{m}, \theta_{-r-m} \sigma_{k}\right) \psi_{k}(-r-m) \stackrel{k \rightarrow \infty}{\longrightarrow} x_{-m+1},
$$


and hence $\tilde{\varphi}_{0}\left(1, \theta_{-r-m} \sigma\right) x_{-m}=x_{-m+1}$ for each $m \geqslant 1$. Consequently the map $\psi_{0}: \mathbb{R} \rightarrow X$ given by

$$
\psi_{0}(t)= \begin{cases}\tilde{\varphi}_{0}\left(t+m, \theta_{-r-m} \sigma\right) x_{-m}, & \text { if } t \in[-m,-m+1], m \geqslant 1, \\ \tilde{\varphi}_{0}\left(t, \theta_{-r} \sigma\right) x_{0}, & \text { if } t \geqslant 0,\end{cases}
$$

is a global solution of $\tilde{\varphi}_{0}$ through $x_{0}$ at $\theta_{-r} \sigma$. It remains to show that $\psi_{0}$ is bounded. In fact, let $s \in \mathbb{R}$ and observe that if $s \geqslant 0$, then $\psi_{0}(s)=\tilde{\varphi}_{0}\left(s, \theta_{-r} \sigma\right) x_{0}$. By Proposition 3.7, there is a sequence $\left\{\gamma_{k}\right\} \subset[0,+\infty)$ such that $\gamma_{k} \stackrel{k \rightarrow \infty}{\longrightarrow} 0$ and

$$
\psi_{k}\left(s+\gamma_{k}-r\right)=\tilde{\varphi}_{\eta_{k}}\left(s+\gamma_{k}, \theta_{-r} \sigma_{k}\right) \psi_{k}(-r) \stackrel{k \rightarrow \infty}{\longrightarrow} \tilde{\varphi}_{0}\left(s, \theta_{-r} \sigma\right) x_{0}=\psi_{0}(s),
$$

and since $\psi_{k}\left(s+\gamma_{k}-r\right) \in \overline{\bigcup \psi_{k}(\mathbb{R})}$ for all $k \geqslant 1$, we have $\psi_{0}(s) \in \overline{\bigcup \psi_{k}(\mathbb{R})}$.

On the other hand, if $s \in[-m,-m+1]$ for some $m \geqslant 1$, we have $\psi_{0}(s)=\tilde{\varphi}_{0}(s+$ $\left.m, \theta_{-r-m} \sigma\right) x_{-m}$. Again, using Proposition 3.7, there exists a sequence $\left\{\alpha_{k}\right\} \subset[0,+\infty)$ such that $\alpha_{k} \stackrel{k \rightarrow \infty}{\longrightarrow} 0$ and

$\psi_{k}\left(-r+s+\alpha_{k}\right)=\tilde{\varphi}_{\eta_{k}}\left(s+m+\alpha_{k}, \theta_{-r-m} \sigma_{k}\right) \psi_{k}(-r-m) \stackrel{k \rightarrow \infty}{\longrightarrow} \tilde{\varphi}_{0}\left(s+m, \theta_{-r-m} \sigma\right) x_{-m}=\psi_{0}(s)$, which implies that $\psi_{0}(s) \in \overline{\bigcup \psi_{k}(\mathbb{R})}$. Therefore, $\psi_{0}$ is a bounded global solution of $\tilde{\varphi}_{0}$ through $x_{0}$ at $\theta_{-r} \sigma$.

Now we suppose, without loss of generality, that $x_{-m} \in M_{0}$ for all $m \geqslant 1$. Let $s_{k}^{m}=$ $\phi_{\eta_{k}}\left(\psi_{k}(-r-m), \theta_{-r-m} \sigma_{k}\right)$ and $r_{k}^{m}=\phi_{\eta_{k}}\left(\psi_{k}(-r-m+1), \theta_{-r-m+1} \sigma_{k}\right)$ for $k \geqslant 1$ and $m \geqslant 1$. By Lemma 4.1 we have $s_{k}^{m} \stackrel{k \rightarrow \infty}{\longrightarrow} 0$ and $r_{k}^{m} \stackrel{k \rightarrow \infty}{\longrightarrow} 0$. As $y_{k, m}=\psi_{k}(-r-m) \stackrel{k \rightarrow \infty}{\longrightarrow} x_{-m} \in M_{0}$ for all $m \geqslant 1$, we obtain

$$
z_{k, m}=\tilde{\varphi}_{\eta_{k}}\left(s_{k}^{m}, \theta_{-r-m} \sigma_{k}\right) y_{k, m} \stackrel{k \rightarrow \infty}{\longrightarrow} I_{0}\left(x_{-m}\right)=z_{0, m} \quad \text { for } m \geqslant 1 .
$$

As $z_{0, m} \notin M_{0}$ because $I_{0}\left(M_{0}\right) \bigcap M_{0}=\varnothing$, it follows by Proposition 3.7 that there exists a sequence $\left\{\eta_{k}^{m}\right\} \subset[0, \infty)$ such that $\eta_{k}^{m} \stackrel{k \rightarrow \infty}{\longrightarrow} 0$ and

$$
\tilde{\varphi}_{\eta_{k}}\left(1+\eta_{k}^{m}, \theta_{-r-m+s_{k}^{m}} \sigma_{k}\right) z_{k, m} \stackrel{k \rightarrow \infty}{\longrightarrow} \tilde{\varphi}_{0}\left(1, \theta_{-r-m} \sigma\right) z_{0, m} \notin M_{0} .
$$

Using Proposition 3.9.

$$
\tilde{\varphi}_{\eta_{k}}\left(1+\eta_{k}^{m}+r_{k}^{m}, \theta_{-r-m+s_{k}^{m}} \sigma_{k}\right) z_{k, m} \stackrel{k \rightarrow \infty}{\longrightarrow} \tilde{\varphi}_{0}\left(1, \theta_{-r-m} \sigma\right) z_{0, m} .
$$

On the other hand, we note that

$$
\begin{aligned}
\tilde{\varphi}_{\eta_{k}}\left(1+\eta_{k}^{m}+r_{k}^{m}, \theta_{-r-m+s_{k}^{m}} \sigma_{k}\right) z_{k, m} & =\tilde{\varphi}_{\eta_{k}}\left(1+\eta_{k}^{m}+r_{k}^{m}+s_{k}^{m}, \theta_{-r-m} \sigma_{k}\right) y_{k, m} \\
& =\tilde{\varphi}_{\eta_{k}}\left(\eta_{k}^{m}+r_{k}^{m}+s_{k}^{m}, \theta_{-r-m+1} \sigma_{k}\right) \tilde{\varphi}_{\eta_{k}}\left(1, \theta_{-r-m} \sigma_{k}\right) y_{k, m} \\
& =\tilde{\varphi}_{\eta_{k}}\left(\eta_{k}^{m}+r_{k}^{m}+s_{k}^{m}, \theta_{-r-m+1} \sigma_{k}\right) \psi_{k}(-r-m+1),
\end{aligned}
$$

for each $k \geqslant 1$. Since $y_{k, m-1}=\psi_{k}(-r-m+1) \stackrel{k \rightarrow \infty}{\longrightarrow} x_{-m+1} \in M_{0}, m \geqslant 2$, we have

$$
\tilde{\varphi}_{\eta_{k}}\left(r_{k}^{m}, \theta_{-r-m+1} \sigma_{k}\right) \psi_{k}(-r-m+1) \stackrel{k \rightarrow \infty}{\longrightarrow} I_{0}\left(x_{-m+1}\right)=z_{0, m-1} \notin M_{0}, \quad m \geqslant 2,
$$


and by Proposition 3.9,

$$
\tilde{\varphi}_{\eta_{k}}\left(\eta_{k}^{m}+r_{k}^{m}+s_{k}^{m}, \theta_{-r-m+1} \sigma_{k}\right) \psi_{k}(-r-m+1) \stackrel{k \rightarrow \infty}{\longrightarrow} I_{0}\left(x_{-m+1}\right)=z_{0, m-1}, \quad m \geqslant 2 .
$$

Hence, $\tilde{\varphi}_{0}\left(1, \theta_{-r-m} \sigma\right) z_{0, m}=z_{0, m-1}$ for all $m \geqslant 2$. Thus the map $\psi_{0}: \mathbb{R} \rightarrow X$ given by

$$
\psi_{0}(t)= \begin{cases}\tilde{\varphi}_{0}\left(t+m, \theta_{-r-m} \sigma\right) z_{0, m}, & \text { if } t \in[-m,-m+1], m \geqslant 2, \\ \tilde{\varphi}_{0}\left(t+1, \theta_{-r-1} \sigma\right) z_{0,1}, & \text { if } t \geqslant-1 .\end{cases}
$$

is a global solution of $\tilde{\varphi}_{0}$ through $\tilde{\varphi}_{0}\left(1, \theta_{-r-1} \sigma\right) z_{0,1}$ at $\theta_{-r} \sigma$.

We claim that $\tilde{\varphi}_{0}\left(1, \theta_{-r-1} \sigma\right) z_{0,1}=x_{0}$. Indeed, note that

$$
\tilde{\varphi}_{0}\left(1, \theta_{-r-1} \sigma\right) z_{0,1}=\tilde{\varphi}_{0}\left(1, \theta_{-r-1} \sigma\right) I_{0}\left(x_{-1}\right) .
$$

By Lemma 4.1, there exists a sequence $\left\{\beta_{k}\right\} \subset[0,1]$ with $\beta_{k} \stackrel{k \rightarrow \infty}{\longrightarrow} 0$ such that

$$
\tilde{\varphi}_{\eta_{k}}\left(\beta_{k}+1, \theta_{-r-1} \sigma_{k}\right) \psi_{k}(-r-1) \stackrel{k \rightarrow \infty}{\longrightarrow} \tilde{\varphi}_{0}\left(1, \theta_{-r-1} \sigma\right) I_{0}\left(x_{-1}\right) .
$$

On the other hand, using Proposition 3.9, we have

$$
\begin{gathered}
\tilde{\varphi}_{\eta_{k}}\left(\beta_{k}+1, \theta_{-r-1} \sigma_{k}\right) \psi_{k}(-r-1)=\tilde{\varphi}_{\eta_{k}}\left(\beta_{k}, \theta_{-r} \sigma_{k}\right) \tilde{\varphi}_{\eta_{k}}\left(1, \theta_{-r-1} \sigma_{k}\right) \psi_{k}(-r-1)= \\
=\tilde{\varphi}_{\eta_{k}}\left(\beta_{k}, \theta_{-r} \sigma_{k}\right) \psi_{k}(-r) \stackrel{k \rightarrow \infty}{\longrightarrow} x_{0} .
\end{gathered}
$$

Thus, $\tilde{\varphi}_{0}\left(1, \theta_{-r-1} \sigma\right) z_{0,1}=x_{0}$. In conclusion $\psi_{0}: \mathbb{R} \rightarrow X$ is a global solution through $x_{0}$ at $\theta_{-r} \sigma$. Using the argument of the previous case, we have $\psi_{0}$ bounded.

$\star$ Proof of $(i i)$. Since $x_{0} \in M_{0}$, if follows by (2.1) that there is $\epsilon_{x_{0}, \sigma}>0$ such that

$$
\bigcup_{t \in\left(0, \epsilon_{x_{0}, \sigma}\right)} F_{\varphi_{0}}\left(x_{0}, t, \theta_{-t} \sigma\right) \bigcap M_{0}=\varnothing .
$$

By Lemma 4.2 , there exists $m_{0} \in \mathbb{N}$ which we may assume $\frac{1}{m_{0}}<\epsilon_{x_{0}, \sigma}$, such that for $m \geqslant m_{0}$ one may find $k_{0}^{m} \in \mathbb{N}$ with

$$
\phi_{\eta_{k}}\left(\psi_{k}(-1 / m-r), \theta_{-1 / m-r} \sigma_{k}\right)>\frac{1}{m} \quad \text { for all } k \geqslant k_{0}^{m} .
$$

Thus for $k \geqslant k_{0}^{m}$ we have

$$
\varphi_{\eta_{k}}\left(\frac{1}{m}, \theta_{-1 / m-r} \sigma_{k}\right) \psi_{k}(-1 / m-r)=\tilde{\varphi}_{\eta_{k}}\left(\frac{1}{m}, \theta_{-1 / m-r} \sigma_{k}\right) \psi_{k}(-1 / m-r)=\psi_{k}(-r) \notin M_{\eta_{k}},
$$

and by 4.2 we may assume that $\psi_{k}(-1 / m-r) \stackrel{k \rightarrow \infty}{\longrightarrow} u_{m}$. As $k \rightarrow \infty$ in (4.5), we get by (H1) that

$$
\varphi_{0}\left(\frac{1}{m}, \theta_{-1 / m-r} \sigma\right) u_{m}=x_{0} \in M_{0} \text { for each } m \geqslant m_{0} .
$$

Moreover, using (4.4), we have $u_{m} \notin M_{0}$ for all $m \geqslant m_{0}$ since $\frac{1}{m}<\epsilon_{x_{0}, \sigma}$.

Now as $\psi_{k}(-1 / m-r) \stackrel{k \rightarrow \infty}{\longrightarrow} u_{m} \notin M_{0}$ for $m \geqslant m_{0}$, it follows by item $(i)$, that there exists a bounded global solution $\psi_{0}^{m}$ of $\tilde{\varphi}_{0}$ through $u_{m}$ at $\theta_{-1 / m-r} \sigma$, for each $m \geqslant m_{0}$. Using (4.2) again, 
we may assume that $\left\{u_{m}\right\}_{m \geqslant m_{0}}$ is convergent and by 4.6 we have $u_{m} \stackrel{m \rightarrow \infty}{\longrightarrow} x_{0}$, which ends the proof.

q.e.d.

Theorem 4.4. Assume that (A1) and (A2) hold. Suppose additionally that for each $\sigma \in \Sigma$ :

(a) $\overline{\bigcup_{\eta \in[0,1]} \bigcup_{t \in \mathbb{R}} A_{\eta}\left(\theta_{t} \sigma\right)}$ compact;

(b) $\lim _{s \rightarrow 0^{+}} \mathrm{d}_{H}\left(A_{0}\left(\theta_{-s} \sigma\right), A_{0}(\sigma)\right)=0$.

Then, given $\sigma \in \Sigma$ and sequences $\left\{\eta_{k}\right\},\left\{\epsilon_{k}\right\} \subset[0,1]$ with $\eta_{k}, \epsilon_{k} \stackrel{k \rightarrow \infty}{\longrightarrow} 0$, we have

$$
\lim _{k \rightarrow \infty} \mathrm{d}_{H}\left(A_{\eta_{k}}\left(\sigma_{k}\right) \backslash M_{\eta_{k}}, A_{0}(\sigma)\right)=0
$$

where $\sigma_{k}=\theta_{\epsilon_{k}} \sigma, k \in \mathbb{N}$.

In particular, for each $\sigma \in \Sigma$, we have

$$
\lim _{k \rightarrow \infty} \mathrm{d}_{H}\left(A_{\eta_{k}}(\sigma) \backslash M_{\eta_{k}}, A_{0}(\sigma)\right)=0 .
$$

Moreover, if $A_{\eta}(\sigma)=\overline{A_{\eta}(\sigma) \backslash M_{\eta}}$ for each $\eta \in[0,1]$ and $\sigma \in \Sigma$, then the family $\left\{\hat{A}_{\eta}\right\}_{\eta \in[0,1]}$ is upper semicontinuous at 0 .

Proof. Let $\sigma \in \Sigma$ be fixed, $\left\{\epsilon_{k}\right\},\left\{\eta_{k}\right\} \subset(0,1]$ and $\left\{x_{k}\right\} \subset X$ be sequences such that $\eta_{k}, \epsilon_{k} \stackrel{k \rightarrow \infty}{\longrightarrow}$ 0 and $x_{k} \in A_{\eta_{k}}\left(\sigma_{k}\right) \backslash M_{\eta_{k}}$ for all $k \geqslant 1$. We shall prove that there exists $x_{0} \in A_{0}(\sigma)$ such that $x_{k} \stackrel{k \rightarrow \infty}{\longrightarrow} x_{0}$, up to a subsequence.

Using condition $(a)$, we can assume that there exists $x_{0} \in X$ such that $x_{k} \stackrel{k \rightarrow \infty}{\longrightarrow} x_{0}$, up to a subsequence. So, it remains to prove that $x_{0} \in A_{0}(\sigma)$.

Since $x_{k} \in A_{\eta_{k}}\left(\sigma_{k}\right) \backslash M_{\eta_{k}}$ for each $k \geqslant 1$, we use the notation of (A2), and condition (a) in particular implies (4.2) (see Corollary 2.18). Moreover, we can assume that

$$
\psi_{k}(-m) \stackrel{k \rightarrow \infty}{\longrightarrow} x_{-m} \text { for each } m \geqslant 1 \text {. }
$$

If $x_{0} \notin M_{0}$, Lemma 4.3 item $(i)$ guarantees that there exists a bounded global solution $\psi_{0}$ for $\tilde{\varphi}_{0}$ at $\sigma$ through $x_{0}$ given by 4.3 , that is, $x_{0} \in A_{0}(\sigma)$.

If $x_{0} \in M_{0}$ then Lemma 4.3 item (ii) implies that there exist $m_{0} \geqslant 1$ such that the sequence $\left\{\psi_{k}(-1 / m)\right\}$ admits a subsequence, denoted by the same, such that $\psi_{k}(-1 / m) \stackrel{k \rightarrow \infty}{\longrightarrow} u_{m} \notin M_{0}$ for $m \geqslant m_{0}$. Moreover, $u_{m} \stackrel{m \rightarrow \infty}{\longrightarrow} x_{0}$ and there exists a bounded global solution $\psi_{0}^{m}$ of $\tilde{\varphi}_{0}$ through $u_{m}$ at $\theta_{-1 / m} \sigma$ for all $m \geqslant m_{0}$, that is, $u_{m} \in A_{0}\left(\theta_{-1 / m} \sigma\right)$ for all $m \geqslant m_{0}$. Hence, using condition $(b)$, we conclude that $x_{0} \in A_{0}(\sigma)$.

The last assertion follows from the identity

$$
\mathrm{d}_{H}(C, D)=\mathrm{d}_{H}(\bar{C}, D) \text { for all nonempty sets } C, D \text {. }
$$




\section{Applications}

We will revisit Example 3.3, to use Theorem 4.4 to obtain upper semicontinuity of the impulsive cocycle attractors. Let $\left[\left(\varphi_{\eta}, \theta\right)_{(\mathbb{R}, \Sigma)}, M_{\eta}, I_{\eta}\right]$ be the impulsive nonautonomous dynamical system defined by (3.2), which satisfies conditions $(\mathrm{H} 1)-(\mathrm{H} 4)$ and the points -1 and 1 of $M_{0} \times \Sigma$ satisfy $\varphi_{0}$-C-SSTC.

Proposition 5.1. There exists a compact subset $K_{0}$ of $\mathbb{R}$ that pullback $\tilde{\varphi}_{\eta}$-absorbs all fixed bounded sets of $\mathbb{R}$, for each $\eta \in[0,1]$. Moreover, if $\mathfrak{D}$ is the universe of all nonautonomous sets $\hat{D}$ with $\bigcup_{g \in \Sigma} D(g)$ bounded in $\mathbb{R}$, the compact nonautonomous set $\hat{K}_{0}=\left\{K_{0}\right\}_{g \in \Sigma}$ is pullback $\left(\tilde{\varphi}_{\eta}, \mathfrak{D}\right)$-absorbing, for each $\eta \in[0,1]$.

Proof. Fix $B$ a bounded subset of $\mathbb{R}$.

If $B \subset[-1-\eta, 1+\eta]$ then $\tilde{\varphi}_{\eta}\left(t, g_{-t}\right) x=\varphi_{\eta}\left(t, g_{-t}\right) x \in[-1-\eta, 1+\eta]$ for all $x \in B, t \geqslant 0$, $\eta \in[0,1]$ and $g \in \Sigma$.

Otherwise, let $b^{*}=\sup |B|=\sup \{|b|: b \in B\}$ and fix $h \in \Sigma$. Note that $s_{\eta}=\phi_{\eta}\left(b^{*}, h\right)<\infty$ since $b^{*}>1+\eta$. Therefore,

$$
\int_{0}^{s_{\eta}} h(u) d u+\eta s_{\eta}=\ln \left(\frac{b^{*}}{1+\eta}\right)
$$

and thus

$$
\int_{0}^{s_{\eta}} h(u) d u \leqslant \ln \left(b^{*}\right) \text { for all } h \in \Sigma \text { and } \eta \in[0,1] .
$$

By (3.1) we get $\int_{0}^{+\infty} h(u) d u=+\infty$ for each $h \in \Sigma$, and thus $\sup _{h \in \Sigma, \eta \in[0,1]} \phi_{\eta}\left(b^{*}, h\right)<\infty$. Let $t^{*}=\sup _{h \in \Sigma, \eta \in[0,1]} \phi_{\eta}\left(b^{*}, h\right)<\infty$.

If $x \in B \bigcap(1+\eta, \infty)$ then $\tilde{\varphi}_{\eta}\left(t, g_{-t}\right) x \in[1+\eta, 2+\eta]$ for all $t \geqslant t^{*}, \eta \in[0,1]$ and $g \in \Sigma$. Analogously, if $x \in B \bigcap(-\infty,-1-\eta)$ then $\tilde{\varphi}_{\eta}\left(t, g_{-t}\right) x \in[-2-\eta,-1-\eta]$ for all $t \geqslant t^{*}, \eta \in[0,1]$ and $g \in \Sigma$.

Thus, taking $K_{0}=[-3,3]$ we have

$$
\tilde{\varphi}_{\eta}\left(t, g_{-t}\right) B \subset K_{0} \quad \text { for all } t \geqslant t^{*} .
$$

Moreover, given a nonautonomous set $\hat{B} \in \mathfrak{D},(5.1)$ also holds, just making $B=\bigcup_{g \in \Sigma} B(g)$, which is bounded in $\mathbb{R}$. q.e.d.

By Theorem 2.16, $\left[\left(\varphi_{\eta}, \theta\right)_{(X, \Sigma)}, M_{\eta}, I_{\eta}\right]$ admits a $\left(\tilde{\varphi}_{\eta}, \mathfrak{D}\right)$-impulsive cocycle attractor $\hat{A}_{\eta}$ for each $\eta \in[0,1]$. Moreover,

$$
\overline{\bigcup_{\eta \in[0,1]} \bigcup_{g \in \Sigma} A_{\eta}(g)} \subset K_{0},
$$

which is compact in $\mathbb{R}$. It is not hard to see that $A_{\eta}(g)=[-2-\eta,-1-\eta] \cup\{0\} \cup[1+\eta, 2+\eta]$ for all $g \in \Sigma$. 
Now it remains to verify condition (b) of Theorem 4.4 to conclude the upper semicontinuity of the family $\left\{\hat{A}_{\eta}\right\}_{\eta \in[0,1]}$ at 0 . To that end we will use Corollary 3.12. Note that in this case $M_{0} \bigcap K_{0}=\{-1,1\}$.

We define the function $\tau: \mathbb{R}_{+} \times \Sigma \rightarrow \mathbb{R}$ given by

$$
\tau(s, h)=\exp \left(\int_{0}^{s} h(u) d u\right) \quad \text { for all } s \geqslant 0 \text { and } h \in \Sigma .
$$

Note that, using (3.1),$\tau(\cdot, h)$ is nondecreasing for each fixed $h \in \Sigma$, and also $\tau \geqslant 1$.

Now fix $t>0, g \in \Sigma$ and take $x=1$ (the case $x=-1$ is analogous). Let $n \geqslant 1$ be such that $2^{n-1}<\tau\left(t, g_{-t}\right) \leqslant 2^{n}$ and define the sequence

$$
x_{k}=\left(\frac{1}{2^{n-1}}+\frac{1}{2^{n+k}}\right) \tau\left(t, g_{-t}\right), \quad \text { for } k \geqslant 1 .
$$

Since $2^{n-1}<\tau\left(t, g_{-t}\right) \leqslant 2^{n}$, we have $2^{n-1}\left(\frac{1}{2^{n-1}}+\frac{1}{2^{n+k}}\right)<x_{k} \leqslant 2^{n}\left(\frac{1}{2^{n-1}}+\frac{1}{2^{n+k}}\right)$, that is, $1+\frac{1}{2^{k+1}}<x_{k} \leqslant 2+\frac{1}{2^{k}}$ for all $k \geqslant 1$. Thus $x_{k} \in(1,3)$ for all $k \geqslant 1$.

Fix $k \geqslant 1$ and let $s_{1}=s_{1}(k)=\phi_{0}\left(x_{k}, g_{-t}\right)$.

Proposition 5.2. Under the previous assumptions, the following properties hold:

(a) $x_{k}=\tau\left(s_{1}, g_{-t}\right)$.

(b) $s_{1}>t$ provided $n=1$.

(c) $s_{1}<t$ provided $n \geqslant 2$.

Proof. (a) Note that

$$
\varphi_{0}\left(s_{1}, g_{-t}\right) x_{k}=1 \Leftrightarrow x_{k}=\exp \left(\int_{0}^{s_{1}} g_{-t}(u) d u\right)=\tau\left(s_{1}, g_{-t}\right) .
$$

(b) If this is not the case then

$$
\tau\left(t, g_{-t}\right) \geqslant \tau\left(s_{1}, g_{-t}\right)=x_{k}=\left(1+\frac{1}{2^{k+1}}\right) \tau\left(t, g_{-t}\right),
$$

which is a contradiction.

(c) If this is not the case then

$$
\tau\left(t, g_{-t}\right) \leqslant \tau\left(s_{1}, g_{-t}\right)=x_{k}=\left(\frac{1}{2^{n-1}}+\frac{1}{2^{n+k}}\right) \tau\left(t, g_{-t}\right)
$$

which is a contradiction since $\frac{1}{2^{n-1}}+\frac{1}{2^{n+k}}<1$ for all $n \geqslant 2$ and $k \geqslant 1$. 
For any $h \in \Sigma$, setting $s^{*}(h)=\phi_{0}(2, h)$, we know that $\tau\left(s^{*}(h), h\right)=2$. Set $h_{0}=g_{-t}$, $t_{0}=s_{0}=0$, recall that $s_{1}=\phi_{0}\left(x_{k}, h_{0}\right)$, and define $t_{1}=s_{1}$. We will also set the following

$$
\left\{\begin{array}{c}
h_{1}=g_{-t+t_{1}}, s_{2}=s^{*}\left(h_{1}\right) \text { and } t_{2}=t_{1}+s_{2}, \\
h_{2}=g_{-t+t_{2}}, s_{3}=s^{*}\left(h_{2}\right) \text { and } t_{3}=t_{2}+s_{3}, \\
\vdots \\
h_{n-1}=g_{-t+t_{n-1}}, s_{n}=s^{*}\left(h_{n-1}\right) \text { and } t_{n}=t_{n-1}+s_{n}, n \geqslant 2 .
\end{array}\right.
$$

Before continuing, note that

$$
\exp \left(\int_{t_{j-1}}^{t_{j}} g_{-t}(u) d u\right)=\exp \left(\int_{0}^{s_{j}} h_{j-1}(u) d u\right)=\tau\left(s_{j}, h_{j-1}\right)=\tau\left(s^{*}\left(h_{j-1}\right), h_{j-1}\right)=2,
$$

for $j=2, \ldots, n$.

Proposition 5.3. For $n \geqslant 2$ it holds $t_{n-1} \leqslant t<t_{n}$.

Proof. First, let us argue by contradiction that $t<t_{n-1}$. Then

$$
\begin{aligned}
& \tau\left(t, g_{-t}\right)<\tau\left(t_{n-1}, g_{-t}\right)=\exp \left(\int_{0}^{t_{n-1}} g_{-t}(u) d u\right)=2^{n-2} \exp \left(\int_{0}^{s_{1}} g_{-t}(u) d u\right) \\
= & 2^{n-2} \tau\left(s_{1}, g_{-t}\right)=2^{n-2} x_{k}=2^{n-2}\left(\frac{1}{2^{n-1}}+\frac{1}{2^{n+k}}\right) \tau\left(t, g_{-t}\right)=\left(\frac{1}{2}+\frac{1}{2^{k+2}}\right) \tau\left(t, g_{-t}\right),
\end{aligned}
$$

which is a contradiction since $\frac{1}{2}+\frac{1}{2^{k+2}}<1$ for all $k \geqslant 1$.

Also, suppose to the contrary that $t \geqslant t_{n}$. Then

$$
\tau\left(t, g_{-t}\right) \geqslant \tau\left(t_{n}, g_{-t}\right)=2^{n-1} x_{k}=2^{n-1}\left(\frac{1}{2^{n-1}}+\frac{1}{2^{n+k}}\right) \tau\left(t, g_{-t}\right)=\left(1+\frac{1}{2^{k+1}}\right) \tau\left(t, g_{-t}\right),
$$

which is a contradiction since $1+\frac{1}{2^{k+1}}>1$ for all $k \geqslant 1$. Then the proof is concluded. q.e.d.

Proposition 5.4. There holds $\tilde{\varphi}_{0}\left(t, \theta_{-t} g\right) x_{k} \stackrel{k \rightarrow \infty}{\longrightarrow} 1$.

Proof. If $n=1$, it follows by Proposition 5.2 that

$$
\tilde{\varphi}_{0}\left(t, g_{-t}\right) x_{k}=\varphi_{0}\left(t, g_{-t}\right) x_{k}=x_{k} \exp \left(-\int_{0}^{t} g_{-t}(u) d u\right)=1+\frac{1}{2^{k+1}} \quad \text { for } k \geqslant 1
$$

and therefore $\tilde{\varphi}_{0}\left(t, g_{-t}\right) x_{k} \stackrel{k \rightarrow \infty}{\longrightarrow} 1$.

Now, let $n \geqslant 2$. By Proposition 5.3, $t_{n-1} \leqslant t<t_{n}$, consequently

$$
\tilde{\varphi}_{0}\left(t, \theta_{-t} g\right) x_{k}=\varphi_{0}\left(t-t_{n-1}, h_{n-1}\right) 2 \text {, }
$$


and hence

$$
\begin{aligned}
\tilde{\varphi}_{0}\left(t, \theta_{-t} g\right) x_{k} & =2 \exp \left(-\int_{0}^{t-t_{n-1}} h_{n-1}(u) d u\right)=2 \exp \left(-\int_{t_{n-1}}^{t} g_{-t}(u) d u\right) \\
& =2 \exp \left(\int_{t_{1}}^{t_{n-1}} g_{-t}(u) d u+\int_{0}^{t_{1}} g_{-t}(u) d u-\int_{0}^{t} g_{-t}(u) d u\right) \\
& =2^{n-1} x_{k} \exp \left(-\int_{0}^{t} g_{-t}(u) d u\right) \\
& =1+\frac{1}{2^{k+1}} \quad \text { for all } k \geqslant 1,
\end{aligned}
$$

that is, $\tilde{\varphi}_{0}\left(t, \theta_{-t} g\right) x_{k} \stackrel{k \rightarrow \infty}{\longrightarrow} 1$.

Thus conditions of Corollary 3.12 are satisfied, and we obtain

$$
\lim _{s \rightarrow 0^{+}} \mathrm{d}_{H}\left(A_{0}\left(g_{-s}\right), A_{0}(g)\right)=0 \quad \text { for each } g \in \Sigma \text {. }
$$

Hence, 4.7 holds for $\left\{\hat{A}_{\eta}\right\}_{\eta \in[0,1]}$, as a direct application of Theorem 4.4. We also have $A_{\eta}(g)=\overline{A_{\eta}(g) \backslash M_{\eta}}$, and hence the family $\left\{\hat{A}_{\eta}\right\}_{\eta \in[0,1]}$ is upper semicontinuous at 0 .

\section{Lower semicontinuity}

In this last section, we establish sufficient conditions to obtain the lower semicontinuity for a family of impulsive cocycle attractors. We will describe the theoretical foundation to achieve our result of lower semicontinuity.

Definition 6.1. Let $\psi^{*}: \mathbb{R} \rightarrow X$ be a global solution of $\tilde{\varphi}$ at $\sigma \in \Sigma$ and set $\Psi^{*}=\psi^{*}(\mathbb{R})$.

(i) The unstable set of $\psi^{*}$ at $\sigma \in \Sigma$ is defined by

$$
\begin{gathered}
W^{u}\left(\psi^{*}\right)(\sigma)=\{z \in X: \\
\text { such that } \left.\lim _{t \rightarrow-\infty} d\left(\psi(t), \Psi^{*}\right)=0\right\} .
\end{gathered}
$$

(ii) The unstable set of $\psi^{*}$ is the nonautonomous set $\hat{W}^{u}\left(\psi^{*}\right)=\left\{W^{u}\left(\psi^{*}\right)(\sigma)\right\}_{\sigma \in \Sigma}$.

(iii) Given $\delta>0$, we also define the local unstable set of $\psi^{*}$ at $\sigma \in \Sigma$ by

$$
\begin{aligned}
& W_{\delta}^{u}\left(\psi^{*}\right)(\sigma)=\{z \in X \text { : there exists a global solution } \psi \text { of } \tilde{\varphi} \text { at } \sigma \text { through } z \\
& \text { such that } \left.d\left(\psi(t), \Psi^{*}\right)<\delta \text { for all } t \leqslant 0 \text { and } \lim _{t \rightarrow-\infty} d\left(\psi(t), \Psi^{*}\right)=0\right\} \text {. }
\end{aligned}
$$

(iv) The local unstable set of $\psi^{*}$ is the nonautonomous set $\hat{W}_{\delta}^{u}\left(\psi^{*}\right)=\left\{W_{\delta}^{u}\left(\psi^{*}\right)(\sigma)\right\}_{\sigma \in \Sigma}$.

Lemma 6.2. If $\psi^{*}$ is a global solution of $\tilde{\varphi}$ then $\hat{W}^{u}\left(\psi^{*}\right)$ is $\tilde{\varphi}$-invariant.

Proof. Fix $t \geqslant 0$ and $\sigma \in \Sigma$. For a given $z \in W^{u}\left(\psi^{*}\right)(\sigma)$, there exists a global solution $\psi$ of $\tilde{\varphi}$ at $\sigma$ such that $\psi(0)=z$ such that $\lim _{s \rightarrow-\infty} d\left(\psi(s), \Psi^{*}\right)=0$. Note that $\tilde{\varphi}(t, \sigma) z=\tilde{\varphi}(t, \sigma) \psi(0)=\psi(t)$. 
Define $\psi_{1}: \mathbb{R} \rightarrow X$ by $\psi_{1}(s)=\psi(s+t)$ for $s \in \mathbb{R}$, which is a global solution of $\tilde{\varphi}$ at $\theta_{t} \sigma$ such that $\psi_{1}(0)=\psi(t)=\tilde{\varphi}(t, \sigma) z$. Hence,

$$
\lim _{s \rightarrow-\infty} d\left(\psi_{1}(s), \Psi^{*}\right)=\lim _{s \rightarrow-\infty} d\left(\psi(s+t), \Psi^{*}\right)=0,
$$

and $\tilde{\varphi}(t, \sigma) z \in W^{u}\left(\psi^{*}\right)\left(\theta_{t} \sigma\right)$.

On the other hand, let $z \in W^{u}\left(\psi^{*}\right)\left(\theta_{t} \sigma\right)$. There exists a global solution $\psi$ of $\tilde{\varphi}$ at $\theta_{t} \sigma$ such that $\psi(0)=z$ and $\lim _{s \rightarrow-\infty} d\left(\psi(t), \Psi^{*}\right)=0$.

We claim that $w=\psi(-t) \in W^{u}\left(\psi^{*}\right)(\sigma)$. Indeed, define $\psi_{2}: \mathbb{R} \rightarrow X$ by $\psi_{2}(s)=\psi(s-t)$. Thus $\psi_{2}$ is a global solution of $\tilde{\varphi}$ at $\sigma$ and $\psi_{2}(0)=\psi(-t)=w$. Also

$$
\lim _{s \rightarrow-\infty} d\left(\psi_{2}(s), \Psi^{*}\right)=\lim _{s \rightarrow-\infty} d\left(\psi(s-t), \Psi^{*}\right)=0
$$

and the proof is complete.

q.e.d.

From the above definitions and Corollary 2.18, we have the following straightforward result.

Lemma 6.3. Fix $\mathfrak{D}$ as the universe of nonautonomous sets with $\bigcup_{\sigma \in \Sigma} D(\sigma)$ bounded in $X$. Let $\left[(\varphi, \theta)_{(X, \Sigma)}, M, I\right]$ be an INDS with a $(\tilde{\varphi}, \mathfrak{D})$-impulsive cocycle attractor $\hat{A}$ such that $I(M) \bigcap M=\varnothing$. If $\psi^{*}$ is a bounded global solution of $\tilde{\varphi}$ at $\sigma$ then $W^{u}\left(\psi^{*}\right)(\sigma) \subset A(\sigma) \backslash M$.

Proof. Let $x \in W^{u}\left(\psi^{*}\right)(\sigma)$. There exists a global solution $\psi$ of $\tilde{\varphi}$ at $\sigma$ through $x$ such that $\lim _{s \rightarrow-\infty} d\left(\psi(s), \Psi^{*}\right)=0$. Since $I(M) \bigcap M=\varnothing$, we have $\psi(\mathbb{R}) \bigcap M=\varnothing$. Since $\Psi^{*}$ is bounded and the INDS has a $(\tilde{\varphi}, \mathfrak{D})$-impulsive cocycle attractor, we obtain $\psi$ is bounded. Therefore, using Corollary 2.18, we conclude that $x=\psi(0) \in A(\sigma) \backslash M$.

q.e.d.

Definition 6.4. We say that two global solutions $\psi_{1}$ and $\psi_{2}$ of $\tilde{\varphi}$ at $\sigma$ are separated in the past if $\limsup _{t \rightarrow-\infty} d\left(\psi_{1}(t), \psi_{2}(t)\right)>0$.

Remark 6.5. If $\psi_{1}$ and $\psi_{2}$ are global solutions of $\tilde{\varphi}$ at $\sigma$ which are not separated in the past, then $\lim _{t \rightarrow-\infty} d\left(\psi_{1}(t), \psi_{2}(t)\right)=0$ and, therefore, $W^{u}\left(\psi_{1}\right)(\sigma)=W^{u}\left(\psi_{2}\right)(\sigma)$.

Let $\mathbb{S}(\sigma)$ be the set of all bounded separated global solutions in the past of $\tilde{\varphi}$ at $\sigma \in \Sigma$.

Lemma 6.6. Let $\left[(\varphi, \theta)_{(X, \Sigma)}, M, I\right]$ be an INDS with a $(\tilde{\varphi}, \mathfrak{D})$-impulsive cocycle attractor $\hat{A}$ such that $I(M) \bigcap M=\varnothing$. Then

$$
A(\sigma) \backslash M=\bigcup_{\psi \in \mathbb{S}(\sigma)} W^{u}(\psi)(\sigma) \text { for each } \sigma \in \Sigma .
$$

Proof. Let $\sigma \in \Sigma$ and $x \in A(\sigma) \backslash M$. By Corollary 2.18, there is a bounded global solution $\psi$ though $x$ at $\sigma$. Thus, $x \in W^{u}(\psi)(\sigma)$ by definition of the unstable set. If $\psi \in \mathbb{S}(\sigma)$ then the proof is finished. Otherwise, there is $\psi_{1} \in \mathbb{S}(\sigma)$ such that $\psi_{1}$ and $\psi$ are not separated in the past, and therefore, $W^{u}\left(\psi_{1}\right)(\sigma)=W^{u}(\psi)(\sigma)$ (see Remark 6.5). Hence, $x \in W^{u}\left(\psi_{1}\right)(\sigma)$. Lemma 6.3 ensures the other inclusion. 
The following result provides sufficient conditions for a type of lower semicontinuity at $\eta=0$ of a family of impulsive cocycle attractors.

Theorem 6.7. Let $\left[\left(\varphi_{\eta}, \theta\right)_{(X, \Sigma)}, M_{\eta}, I_{\eta}\right]$ be an INDS with a $\left(\tilde{\varphi}_{\eta}, \mathfrak{D}\right)$-impulsive cocycle attractor $\hat{A}_{\eta}$, for each $\eta \in[0,1]$, satisfying the conditions of Theorem 4.4. Additionally, assume that:

(a) for each $\eta \in[0,1]$ and $\sigma \in \Sigma$ there exists a sequence of separated global solutions in the past $\mathbb{S}_{\eta}(\sigma)=\left\{\psi_{j, \eta}^{*}\right\}_{j \geqslant 1}$ with

$$
\sup _{t \in \mathbb{R}}\left[d\left(\psi_{j, \eta}^{*}(t), \psi_{j, 0}^{*}(t)\right)+d\left(\psi_{j, 0}^{*}(t), \psi_{j, \eta}^{*}(t)\right)\right] \stackrel{\eta \rightarrow 0}{\rightarrow} 0
$$

for each $j \geqslant 1$;

(b) for each $\sigma \in \Sigma$ there exists $\delta>0$ such that

$$
d_{H}\left(W_{\delta}^{u}\left(\psi_{j, 0}^{*}\right)(\sigma), W_{\delta}^{u}\left(\psi_{j, \eta}^{*}\right)(\sigma)\right)+d_{H}\left(W_{\delta}^{u}\left(\psi_{j, \eta}^{*}\right)(\sigma), W_{\delta}^{u}\left(\psi_{j, 0}^{*}\right)(\sigma)\right) \stackrel{\eta \rightarrow 0}{\rightarrow} 0,
$$

for each $j \geqslant 1$;

(c) for each $\sigma \in \Sigma$ we have

$$
A_{0}(\sigma)=\overline{\bigcup_{j \geqslant 1} W^{u}\left(\psi_{j, 0}^{*}\right)(\sigma)}
$$

Then given $\sigma \in \Sigma$ and $x \in A_{0}(\sigma)$ there exist sequences $\eta_{k}, \epsilon_{k} \stackrel{k \rightarrow \infty}{\longrightarrow} 0$ and $x_{k} \in A_{\eta_{k}}\left(\theta_{\epsilon_{k}} \sigma\right) \backslash M_{\eta_{k}}$ such that $x_{k} \stackrel{k \rightarrow \infty}{\longrightarrow} x$.

Proof. Fix $\sigma \in \Sigma, x_{0} \in A_{0}(\sigma)$ and $\epsilon>0$. Using item (c), there exists, $x_{\epsilon} \in \bigcup_{j \geqslant 1} W^{u}\left(\psi_{j, 0}^{*}\right)(\sigma)$ such that

$$
d\left(x_{\epsilon}, x_{0}\right)<\frac{\epsilon}{2} .
$$

Let $j \geqslant 1$ be such that $x_{\epsilon} \in W^{u}\left(\psi_{j, 0}^{*}\right)(\sigma)$ and $\xi_{j}$ be the global solution of $\tilde{\varphi}_{0}$ at $\sigma$ such that $\xi_{j}(0)=x_{\epsilon}$ and $\lim _{t \rightarrow-\infty} d\left(\xi_{j}(t), \Psi_{j, 0}^{*}\right)=0$. There exists $\tau>0$ such that $z_{\epsilon}=\xi_{j}(-\tau) \in$ $W_{\delta}^{u}\left(\psi_{j, 0}^{*}\right)\left(\theta_{-\tau} \sigma\right)$. By condition (b), there exist a sequence $\eta_{k} \stackrel{k \rightarrow \infty}{\longrightarrow} 0$ and $z_{k} \in W_{\delta}^{u}\left(\psi_{j, \eta_{k}}^{*}\right)\left(\theta_{-\tau} \sigma\right)$ such that $z_{k} \stackrel{k \rightarrow \infty}{\longrightarrow} z_{\epsilon}$.

Now, since $z_{\epsilon} \notin M_{0}$ by Lemma 6.6, using Proposition 3.7 there exists a sequence $\left\{\epsilon_{k}\right\} \subset$ $[0,+\infty)$ such that $\epsilon_{k} \stackrel{k \rightarrow \infty}{\longrightarrow} 0$ and

$$
x_{k}:=\tilde{\varphi}_{\eta_{k}}\left(\epsilon_{k}+\tau, \theta_{-\tau} \sigma\right) z_{k} \stackrel{k \rightarrow \infty}{\longrightarrow} \tilde{\varphi}_{0}\left(\tau, \theta_{-\tau} \sigma\right) z_{\epsilon}=\tilde{\varphi}_{0}\left(\tau, \theta_{-\tau} \sigma\right) \xi_{j}(-\tau)=\xi_{j}(0)=x_{\epsilon} .
$$

Then the result follows, noting that $x_{k} \in A_{\eta_{k}}\left(\theta_{\epsilon_{k}} \sigma\right) \backslash M_{\eta_{k}}$.

q.e.d.

Consequently, we obtain the following lower semicontinuity result, with direct proof.

Corollary 6.8. Assume that all the conditions of Theorem 6.7 are satisfied. Suppose also that given sequences $\eta_{k}, \epsilon_{k} \stackrel{k \rightarrow \infty}{\longrightarrow} 0$ and $x_{k} \in A_{\eta_{k}}\left(\theta_{\epsilon_{k}} \sigma\right) \backslash M_{\eta_{k}}$ there exists $z_{k} \in A_{\eta_{k}}(\sigma)$ for each $k \geqslant 1$ such that $d\left(x_{k}, z_{k}\right) \stackrel{k \rightarrow \infty}{\longrightarrow} 0$. Then the family $\left\{\hat{A}_{\eta}\right\}_{\eta \in[0,1]}$ is lower semicontinuous at 0 . 


\section{Conclusion and final remarks}

In this work, we presented the collective tube conditions for nonautonomous impulsive dynamical systems and we proved a result containing sufficient conditions to ensure the upper semicontinuity of impulsive cocycle attractors of nonautonomous dynamical systems. Our main result is Theorem 4.4

The difficulties of this theory appear in the applications: the conditions are very hard to verify, and in most examples are assumed artificially. Even conditions (2.1) for the autonomous case are not easy to verify in examples, both in finite or infinite dimensional spaces (see the application in Section 5).

In this paper we chose to present a more simple example (an ODE in the real line) precisely to show the computations required to verify all the needed conditions. Thus, in the theory of impulsive systems, this is one of the many problems yet to be solved: to find concrete examples in which the conditions can be verified, and also try to find results that simplify the verification of these conditions.

Lastly, we presented abstract results to obtain lower semicontinuity of impulsive cocycle attractors for nonautonomous dynamical systems. As for applications, there is still a large theory to be developed, namely, the study of the unstable sets of invariants and their continuity with respect to small perturbations, and we plan to analyze them in the future.

\section{References}

[1] Aragão-Costa, E. R., Caraballo, T., Carvalho, A. N., \& Langa, J. A.: Nonautonomous Morse decomposition and Lyapunov functions for dynamically gradient processes, Trans. Amer. Math. Soc., 365 (10) (2013), 5277-5312.

[2] Arrieta, J. M., Carvalho A. N. \& Rodríguez-Bernal, A.: Upper semicontinuity of attractors for parabolic problems with nonlinear boundary conditions and localized large diffusion, J. Differential Equations, 168(1) (2000), 33-59.

[3] Bonotto, E. M., Bortolan, M. C., Caraballo, T. \& Collegari, R.: Impulsive nonautonomous dynamical systems and impulsive cocycle attractors, Math. Meth. Appl. Sci., 40 (2017), 1095-1113.

[4] Bonotto, E. M., Bortolan, M. C., Czaja, R \& Collegari, R.: Semicontinuity of attractors for impulsive dynamical systems, J. Differential Equations, 261 (2016), 4338-4367.

[5] Bonotto, E. M., Bortolan, Caraballo, T. \& Collegari, R.: Attractors for impulsive nonautonomous dynamical systems and their relations, J. Differential Equations, 262 (2017), 3524-3550.

[6] Bortolan, M. C., Caraballo, T., Carvalho, A. N. \& Langa, J. A.: Skew product semiflows and Morse decomposition, J. Differential Equations, 255 (2013), 2436-2462.

[7] Caraballo, T. \& Langa, J. A.: On the upper semicontinuity of cocycle attractors for nonautonomous and random dynamical systems, Dynamics of Continuous and Discrete Impulsive Systems - Series A Mathematical Analysis, 10 (2003), 491-513. 
[8] Carvalho, A.N. \& Hines, G.: Lower semicontinuity of attractors for gradient systems, Dynam. Systems Appl., 9(1) (2000), 37-50.

[9] Carvalho, A. N. \& Langa, J. A.: An extension of the concept of gradient semigroups which is stable under perturbation, J. Differential Equations, 246 (2009), 2646-2668.

[10] Carvalho, A. N., Langa, J. A., Robinson, J. C., \& Suárez, A.: Characterization of nonautonomous attractors of a perturbed gradient system, J. Differential Equations, 236 (2007), 570-603.

[11] Carvalho, A. N., Langa, J. A. \& Robinson, J. C.: Lower semicontinuity of attractors for nonautonomous dynamical systems, Ergodic Theory Dynam. Systems, 29(6) (2009), 1765-1780.

[12] Carvalho, A. N., Langa, J. A., \& Robinson, J. C.: Attractors for infinite-dimensional nonautonomous dynamical systems, Applied Mathematical Series 182, Springer, New York (2013).

[13] Chepyzhov, V. V. \& Vishik, M. I.: Attractors for Equations of Mathematical Physics. Colloquium Publications 49, American Mathematical Society, (2002).

[14] Ciesielski, K.: On semicontinuity in impulsive dynamical systems, Bull. Polish Acad. Sci. Math., 52 (2004), $71-80$.

[15] Ciesielski, K.: Sections in semidynamical systems, Bull. Polish Acad. Sci. Math., 40 (1992), 297-307.

[16] Hale, J.K. \& Raugel, G.: Lower semicontinuity of attractors of gradient systems and applications, Ann. Mat. Pura Appl., 154 (4) (1989), 281-326.

[17] Hale, J.K. \& Raugel, G.: Lower semicontinuity of the attractor for a singularly perturbed hyperbolic equation. J. Dynam. Differential Equations, 2 (1990), 19-67.

[18] Kloeden, P. E. \& Rasmussen, M.: Nonautonomous Dynamical Systems, AMS Mathematical Surveys and Monographs, (2011).

E. M. Bonotto, Instituto de Ciências Matemáticas e de Computação, Universidade de São Paulo, Campus de São Carlos, Caixa Postal 668, São Carlos, SP, Brazil.

E-mail adress, E. M. Bonotto: ebonotto@icmc.usp.br

M. C. Bortolan, Departamento de Matemática, Universidade Federal de Santa Catarina, Campus Trindade, 88040-900, Florianópolis, Brazil.

E-mail adress, M. C. Bortolan: m.bortolan@ufsc.br

T. Caraballo, Departamento de Ecuaciones Diferenciales y Análisis Numérico EdAN, UniverSidad de Sevilla, Sevilla, Spain.

E-mail adress, T. Caraballo: caraball@us.es

R. Collegari, Faculdade de Matemática, Universidade Federal de Uberlândia, 38408-100, Uberlândia, MG, BRAZIL.

E-mail adress, R. Collegari: collegari@gmail.com 